\title{
Frequency-domain calculation of the self force: The high-frequency problem and its resolution
}

\author{
Leor Barack ${ }^{1}$, Amos Ori $^{2}$ and Norichika Sago ${ }^{1}$ \\ ${ }^{1}$ School of Mathematics, University of Southampton, Southampton, SO17 1BJ, United Kingdom \\ ${ }^{2}$ Department of Physics, Technion-Israel Institute of Technology, Haifa, 32000, Israel
}

(Dated: June 26, 2018)

\begin{abstract}
The mode-sum method provides a practical means for calculating the self force acting on a small particle orbiting a larger black hole. In this method, one first computes the spherical-harmonic $l$-mode contributions $F_{l}^{\mu}$ of the "full force" field $F^{\mu}$, evaluated at the particle's location, and then sums over $l$ subject to a certain regularization procedure. In the frequency-domain variant of this scheme the quantities $F_{l}^{\mu}$ are obtained by fully decomposing the particle's self field into Fourierharmonic modes $l m \omega$, calculating the contribution of each such mode to $F_{l}^{\mu}$, and then summing over $\omega$ and $m$ for given $l$. This procedure has the advantage that one only encounters ordinary differential equations. However, for eccentric orbits, the sum over $\omega$ is found to converge badly at the particle's location. This problem (reminiscent of the familiar Gibbs phenomenon of Fourier analysis) results from the discontinuity of the time-domain $F_{l}^{\mu}$ field at the particle's worldline. Here we propose a simple and practical method to resolve this problem. The method utilizes the homogeneous modes $\operatorname{lm} \omega$ of the self field to construct $F_{l}^{\mu}$ (rather than the inhomogeneous modes, as in the standard method), which guarantees an exponentially-fast convergence to the correct value of $F_{l}^{\mu}$, even at the particle's location. We illustrate the application of the method with the example of the monopole scalar-field perturbation from a scalar charge in an eccentric orbit around a Schwarzschild black hole. Our method, however, should be applicable to a wider range of problems, including the calculation of the gravitational self-force using either Teukolsky's formalism, or a direct integration of the metric perturbation equations.
\end{abstract}

\section{INTRODUCTION}

The problem of calculating the gravitational self-force [1, 2, 3] acting on a pointlike test particle as it moves in orbit around a black hole is attracting considerable attention in recent years [4, 5]. Within this context, various authors have been studying also the analogous problem of the scalar-field self-force [6] acting on a particle endowed with a scalar charge, which proved to be a useful toy model. The electromagnetic (EM) self-force, acting on an electric point charge, was also studied by various authors following the seminal work by DeWitt and Brehme [7]. A practical algorithm, commonly used for computing the self force in all three cases (gravitational, EM and scalar), is the mode-sum method [8, 9, 10]. This method requires as input the multipole modes of the full (retarded) perturbation fields, along with their derivatives, evaluated at the particle's location. These multipoles can be calculated using either frequencydomain methods (as in, e.g., [11, 12, 13]) or time-domain numerical evolution (as in, e.g., 14, 15, 16, 17]). In both computational approaches one sets off by writing down the appropriate set of perturbation equations, modeling the source term associated with the point particle (namely, the energy-momentum, the electric four-current, or the scalar charge) as a delta-function distribution. In the frequency-domain approach one then decomposes the inhomogeneous perturbation equations into Fourier-harmonic modes ("lmw modes") and proceeds by solving the resulting ordinary differential equations (ODEs) with suitable boundary conditions at spatial infinity and at the event horizon. In the alternative, time-domain approach, one refrains from decomposing the field into frequency modes, and instead tackles the partial differential equations for each $l, m$ directly using time evolution.

Each of the above two approaches has its strengths and weaknesses. The time-domain approach has the advantage that one only deals with a single field for each $l m$, whereas in the frequency-domain approach one has to sum over the various $\omega$ modes. On the other hand, the latter approach has the obvious advantage that one only faces ODEs. Despite the fact that time-domain methods are winning growing popularity in recent years, frequency-domain calculations remain an appealing option for some range of orbital parameters [18]. Also, it turns out that the non-radiative multipoles of the gravitational perturbation in Schwarzschild (i.e., the modes $l=0,1$ ) are difficult to analyze in the time domain, due to instabilities [15, 17], and one resorts to a frequency-domain calculation in this case [19]. Working in the frequency domain, however, brings about a technical issue which, to the best of our knowledge, has not been addressed so far in the current context.

To illustrate the problem, is it instructive to refer to the simple case of (minimally-coupled and massless) scalarfield perturbations from a pointlike scalar charge orbiting a Schwarzschild black hole. In this case, the scalar field $\Phi(t, r, \theta, \varphi)$ can be decomposed in spherical harmonics $Y_{l m}(\theta, \varphi)$, yielding the multipolar mode functions $\phi_{l m}(t, r)$. Here $t, r, \theta, \varphi$ are the standard Schwarzschild coordinates. Let us denote the $r$ value of the particle's location at time $t$ by $r_{\mathrm{p}}(t)$. With suitable boundary/initial conditions, a unique solution is obtained for $\phi_{l m}$ (for each $l, m$ ), which is 
continuous along $r=r_{\mathrm{p}}(t)$. However, the derivatives $\phi_{l m, r}$ and $\phi_{l m, t}$ will generally suffer a finite jump discontinuity across $r=r_{\mathrm{p}}(t)$, which reflects the presence of a source "shell" resulting from decomposing the point charge in spherical harmonics. In particular, if the orbit is eccentric, the derivatives of $\phi_{l m}$ will generally be discontinuous functions of $t$ at a fixed value of $r$ along the orbit.

Now imagine trying to reconstruct $\phi_{l m}(t, r)$ (for some fixed $r$ along the orbit) as a sum over its Fourier components:

$$
\phi_{l m}(t, r)=\sum_{\omega} R_{l m \omega}(r) e^{-i \omega t} .
$$

Since, for an eccentric orbit, $\phi_{l m}(t, r)$ is only a $C^{0}$ function of $t$ at the particle's worldline, it follows from standard Fourier theory [20] that the Fourier sum in Eq. (11) will only converge there like $\sim \omega^{-1}$. The actual situation is even worse, however, because for self-force calculations we need not only $\phi_{l m}(t, r)$ but also its derivatives. For instance, to calculate the $r$ component of the self force we need to evaluate (one-sided limits of) $\phi_{l m, r}(t, r)$ as $r$ approaches $r_{\mathrm{p}}(t)$. Suppose again that we want to reconstruct this quantity from its Fourier components (namely the functions $R_{l m \omega, r}$ ). Since $\phi_{l m, r}(t, r)$ is a discontinuous function of $t$, we will inevitably face here the well known "Gibbs phenomenon" [21]. Namely, the Fourier sum will fail to converge to the right value at $r \rightarrow r_{\mathrm{p}}(t)$. (The problematic behavior of the Fourier sum is simply a consequence of our attempt to construct a discontinuous function - or a non-smooth function in the case of the field $\phi_{l m}$ itself-from a sum over smooth harmonics.)

From a practical point of view this would mean that (i) at the coincidence limit $r \rightarrow r_{\mathrm{p}}(t)$ the sum over $\omega$ modes would fail to yield the correct one-sided values of $\phi_{l m, r}(t, r)$, however many $\omega$ modes are included in the sum; and (ii) if we reconstruct $\phi_{l m, r}$ at a point $r=r_{0}$ off the worldline, then the Fourier series should indeed converge; Alas, the number of $\omega$ modes required for achieving a prescribed precision would grow unboundedly as $r_{0}$ approaches $r_{\mathrm{p}}(t)$, making it extremely difficult to evaluate $\phi_{l m, r}$ at the coincidence limit.

This technical difficulty is rather generic, and will show also in calculations of the local EM and gravitational fields. Consider, as a second example, the gravitational perturbation from a point mass moving in an eccentric orbit in Schwarzschild: In suitable gauges (like the Lorenz gauge, often applied in self-force calculations) the multipole $l m$ modes of the physical metric perturbation [26] are again $C^{0}$ functions of $r$ and $t$ along the orbit, and their derivatives are generally discontinuous there. Attempting to construct them naively from a sum over frequency modes would encounter the same difficulty as in the scalar case: A poor convergence of the ( $l m$-modes of the) metric perturbations, and lack of convergence for their derivatives.

For orbits in Kerr spacetime the situation is basically similar though more subtle. The Kerr variant of the modesum method [10] requires, just as in its Schwarzschild counterpart, the spherical harmonic modes $\phi_{l m}(r, t)$ of the perturbation field (as well as their derivatives) as input. 27] For given $l m$, the spherical-harmonic decomposition of the point charge will again result in a $\delta$-function-type source term distributed over a shell, which in turn renders the derivatives of $\phi_{l m}(r, t)$ discontinuous. Therefore an attempt to construct $\phi_{l m}(r, t)$ (and, more crucially, its derivatives) through a naive summation over its Fourier modes will lead to the same difficulties as in the Schwarzschild case.

The problem discussed here takes an even more extreme form when considering EM or gravitational perturbations via the Teukolsky formalism: Here, the $l m$ modes of the perturbation fields (now the Newman-Penrose fields $\varphi_{0}, \varphi_{2}$ or $\left.\Psi_{0}, \Psi_{4}\right)$ are not even continuous at the particle's orbit - a consequence of the fact that the source term for Teukolsky's equation involves derivatives of the electric four-current or the energy-momentum tensor associated with the particle (a single derivative in the EM case; a second derivative in the gravitational case). [28] Again, a naive attempt to construct these multipoles as a sum over their $\omega$ modes will be hampered by the Gibbs phenomenon, and the associated lack of convergence.

In this article we propose a way around the above problem, which is both elegant and extremely simple. In our method we use the homogeneous radial functions $R_{\operatorname{lm} \omega}(r)$ (extended all the way through to the particle's worldline), instead of the actual inhomogeneous functions. The Fourier sum of these homogeneous radial functions is found to converge exponentially-fast, and to yield the correct values of the perturbation multipoles (and their derivatives) along the particle's worldline. We shall focus in this paper on the scalar-field case. We justify our new method using simple analytical arguments, and then demonstrate the validity of the method (and the exponential convergence) with an explicit numerical calculation in the case $l=0$. The same method should be applicable, however, for any of the other problems mentioned above: EM and gravitational perturbations using Teukolsky's equation (or SasakiNakamura's equation), as well as metric perturbations in the Lorenz gauge. A forthcoming paper [19] will report on the computation of the local monopole and dipole modes of the Lorenz-gauge perturbation (for eccentric orbits in Schwarzschild), facilitated by the new method suggested here.

This paper is structured as follows. In Sec. II we set up the physical scenario-a pointlike scalar charge in a bound orbit around a Schwarzschild black hole - and review the formalism commonly used in this case to construct the scalar-field multipoles and the scalar self-force. Section III demonstrates how the naive sum over frequencies leads to the Gibbs phenomenon and to the associated problematic convergence at the particle's location. Then in Sec. IV we present our new method of extended homogeneous solutions, and show how it cures the problematic behavior 
of the Fourier sum. We provide the theoretical justification to this method, as well as numerical verification in the monopole $(l=0)$ case. In Sec. V we highlight the advantages of the new method and discuss foreseeable applications. Appendices $\mathrm{A}$ C give details of the methods used for our numerical illustrations, and App. D contains some technical details relating to the formal justification of our new method.

Throughout this work we use standard geometrized units (with $c=G=1$ ) and metric signature $(-+++)$.

\section{PRELIMINARIES}

\section{A. Physical setup and scalar-field equation}

Consider a pointlike particle which moves on an eccentric, bound geodesic orbit around a Schwarzschild black hole with mass parameter $M$. The particle's worldline is denoted $x_{\mathrm{p}}^{\mu}(\tau)$, where $\tau$ is the proper time. The particle's trajectory is bounded within the range $r_{\min } \leq r \leq r_{\max }$ for certain $r_{\max }$ and $r_{\min }>4 M$. Without loss of generality we shall take the orbit to be equatorial, namely $\theta_{\mathrm{p}}=\pi / 2$.

Assume now that the particle carries a scalar charge $q$. This charge couples to a massless, minimally-coupled scalar field $\Phi\left(x^{\mu}\right)$, satisfying the field equation

$$
\square \Phi=-4 \pi \rho
$$

Here $\rho$ is the scalar charge density, which takes the form of a $\delta$-function along the particle's worldline:

$$
\rho(x)=q \int_{-\infty}^{\infty} \delta^{4}\left[x-x_{\mathrm{p}}(\tau)\right][-g(x)]^{-1 / 2} d \tau,
$$

where $g=-r^{4} \sin ^{2} \theta$ is the metric determinant, and hereafter the vectorial indices of $x^{\mu}(\tau)$ and $x_{\mathrm{p}}^{\mu}(\tau)$ are suppressed for brevity.

Since $t$ is timelike (hence monotonic) we can use it instead of $\tau$ to parametrize the orbit. In the $r, t$ plane the orbit is then denoted by $r=r_{\mathrm{p}}(t)$. Transforming the integration variable in Eq. (3) from $\tau$ to $t$ and substituting $\theta_{\mathrm{p}}=\pi / 2$, we find

$$
\rho=q\left(r^{2} u^{t}\right)^{-1} \delta\left[r-r_{\mathrm{p}}(t)\right] \delta\left[\varphi-\varphi_{\mathrm{p}}(t)\right] \delta[\theta-\pi / 2],
$$

where $u^{t} \equiv d t_{\mathrm{p}} / d \tau$. Note that $u^{t}$ only depends on $r_{\mathrm{p}}(t)$ : We have $u^{t}=E\left[1-2 M / r_{\mathrm{p}}(t)\right]^{-1}$, where $E$ is a constant of motion.

\section{B. Spherical-harmonics decomposition}

We now separate the field equation (2) by decomposing $\Phi$ in spherical harmonics, in the form

$$
\Phi=\sum_{l=0}^{\infty} \sum_{m=-l}^{l} \phi_{l m}(t, r) Y_{l m}(\theta, \varphi) / r .
$$

Here $Y_{l m}$ are the standard (complex-valued) normalized spherical harmonics given by $Y_{l m}=c_{l m} P_{l m}(\cos \theta) e^{i m \varphi}$, where $P_{l m}$ are the associated Legendre polynomials and $c_{l m}$ are (real) normalization constants. The factor $1 / r$ is introduced for later convenience. The charge density in Eq. (4) is decomposed in a similar manner:

$$
\rho=\sum_{l=0}^{\infty} \sum_{m=-l}^{l} \hat{\rho}_{l m}(t, r) Y_{l m}(\theta, \varphi)
$$

where

$$
\hat{\rho}_{l m}(t, r)=\int \rho Y_{l m}^{*} d \Omega=\hat{c}_{l m} q\left(r^{2} u^{t}\right)^{-1} e^{-i m \varphi_{\mathrm{p}}(t)} \delta\left[r-r_{\mathrm{p}}(t)\right] .
$$

Here $\hat{c}_{l m}=c_{l m} P_{l m}(0), d \Omega=\sin \theta d \theta d \varphi$ is an element of solid angle, and an asterisk denotes complex conjugation. The field equation (2) is now separated, and for each $l m$ the function $\phi_{l m}(t, r)$ satisfies the partial differential equation

$$
\frac{\partial^{2} \phi_{l m}}{\partial r_{*}^{2}}-\frac{\partial^{2} \phi_{l m}}{\partial t^{2}}-V_{l}(r) \phi_{l m}=-4 \pi r f(r) \hat{\rho}_{l m}
$$


where $f(r) \equiv 1-2 M / r, r_{*} \equiv r+2 M \ln [r /(2 M)-1]$ is the tortoise radial coordinate, and

$$
V_{l}(r)=f(r)\left(\frac{2 M}{r^{3}}+\frac{l(l+1)}{r^{2}}\right) .
$$

The function $\phi_{l m}(t, r)$ is determined for each $l m$ by Eq. (8), supplemented with suitable boundary conditions at null infinity (no incoming waves) and at the event horizon (no outgoing waves). Since the source term has a $\delta$-function form, the function $\phi_{l m}(t, r)$ must be continuous at $r=r_{\mathrm{p}}(t)$, but it will generally fail to be differentiable there: Its $r$ derivative (and also its $t$ derivative, except at the two orbital turning points) will suffer a discontinuity along the orbit. On each side of the worldline $r=r_{\mathrm{p}}(t)$, however, the function $\phi_{l m}(t, r)$ satisfies the homogeneous part of Eq. (8), and, since the homogeneous field equation and the curve $r=r_{\mathrm{p}}(t)$ are both analytic, we expect $\phi_{l m}(t, r)$ to be analytic (in both $r$ and $t$ ) anywhere off the worldline. We shall assume this analyticity here, although we are not aware of a proof. The alternative option appears highly unlikely, because it would mean that the actual field produced by the point charge somehow develops irregularities in the vacuum region off the particle.

\section{Self force via mode sum}

Once the functions $\phi_{l m}$ have been determined (e.g. numerically), the self force acting on the scalar charge may be constructed by the mode-sum method. This procedure is described in detail in Refs. [8, 9, [10]. Here we outline it briefly, in order to provide some perspective on how the quantities $\phi_{l m}$ are incorporated in the construction of the self force.

Let $\Phi_{l}$ denote the contribution of an individual $l$ to $\Phi$ :

$$
\Phi_{l}(t, r, \theta, \varphi)=\sum_{m=-l}^{l} \phi_{l m}(t, r) Y_{l m}(\theta, \varphi) / r .
$$

The $l$-component of the full-force field, $F_{l \mu}(x)$, is then obtained by applying a certain linear differential operator $\mathcal{F}_{\mu}$ to $\phi_{l} . \quad\left[\mathcal{F}_{\mu}\right.$ is the same differential operator which determines the force that a smooth, "non-self" field $\Phi(x)$ would exert on a test charge.] In the scalar-field case we have $\mathcal{F}_{\mu}=q \partial_{\mu}$, and therefore [29]

$$
F_{l \mu}=\mathcal{F}_{\mu} \Phi_{l}=q \sum_{m=-l}^{l} \partial_{\mu}\left[\phi_{l m}(t, r) Y_{l m}(\theta, \varphi) / r\right] .
$$

The quantities $F_{l \mu}$ are to be evaluated at the particle's location. To be more specific, let us denote by $x_{f}$ the event (on the particle's worldline) at which the self force is to be evaluated. Then the quantities $F_{l \mu}$ are to be evaluated at $x \rightarrow x_{f}$ (a somewhat rough statement which will be refined shortly). From Eq. (11) it is obvious that $F_{l \mu}$ depends linearly on the following functions of $r$ and $t$ (for each $m$ ): $\phi_{l m}, \phi_{l m, r}$, and $\phi_{l m, t}$. We use the symbol $\phi_{l m}^{i}(i=0,1,2)$ as an abbreviated notation for these three key functions.

As was discussed above, the quantities $\phi_{l m, r}$ and $\phi_{l m, t}$ are not truly defined on the curve $r=r_{\mathrm{p}}(t)$, and in particular at $x=x_{f}$. Instead, each of these functions has two well-defined (but generally different) one-sided limits, corresponding to approaching the worldline point $x_{f}$ from the range $r>r_{\mathrm{p}}(t)$ or $r<r_{\mathrm{p}}(t)$. We denote these two one-sided limits as $x \rightarrow x_{f+}$ and $x \rightarrow x_{f-}$, respectively. Correspondingly, the quantities $F_{l \mu}$ will each have two one-sided limits, $F_{l \mu}^{+}$and $F_{l \mu}^{-}$, defined by

$$
F_{l \mu}^{ \pm}\left(x_{f}\right) \equiv \lim _{x \rightarrow x_{f \pm}} F_{l \mu}(x)
$$

The self force at $x=x_{f}$ may now be derived from either set of quantities, $F_{l \mu}^{+}$or $F_{l \mu}^{-}$, via the mode-sum formula [ []

$$
F_{\mu}^{\text {self }}=\sum_{l=0}^{\infty}\left[F_{l \mu}^{ \pm}\left(x_{f}\right) \mp(l+1 / 2) A_{\mu}-B_{\mu}\right],
$$

where $A_{\mu}$ and $B_{\mu}$ are certain parameters ("regularization parameter") which Refs. 9, 10] determine analytically. Note that the two one-sided limits in Eq. (13) yield the same value of $F_{\mu}^{\text {self }}$. 


\section{Frequency-domain analysis}

The partial differential equation (8), which determines the functions $\phi_{l m}(t, r)$, may be tackled in either the time domain or the frequency domain. In time-domain calculations, one directly integrates this equation numerically, using time-evolution on a two-dimensional grid. In the frequency-domain method, on the other hand, one first further separates this equation into Fourier frequency modes, using

$$
\phi_{l m}(t, r)=\int d \omega R_{l m \omega}(r) e^{-i \omega t}
$$

and

$$
-4 \pi r f(r) \hat{\rho}_{l m}(t, r)=\int d \omega Z_{l m \omega}(r) e^{-i \omega t} .
$$

Equation (8) then reduces to the ordinary differential equation

$$
\frac{d^{2} R_{l m \omega}}{d r_{*}^{2}}-\left[V_{l}(r)-\omega^{2}\right] R_{l m \omega}=Z_{l m \omega} .
$$

Since $\hat{\rho}_{l m}(t, r)$ only has support on the curve $r=r_{\mathrm{p}}(t)$, it follows that $Z_{l m \omega}(r)$ is only supported within the range $r_{\min } \leq r \leq r_{\max }$.

From Eq. (7) it is evident that $\hat{\rho}_{l m}$ only depends on $t$ through $r_{\mathrm{p}}(t)$ and $\varphi_{\mathrm{p}}(t)$. For an eccentric geodesic $r_{\mathrm{p}}(t)$ is periodic, and $\varphi_{\mathrm{p}}(t)$ also has its inherent $2 \pi$ periodicity. It then follows (see App. D) that $\hat{\rho}_{l m}$ is 2-periodic in $t$, namely it has a discrete spectrum of the form $\omega=n \Omega_{r}+m \Omega_{\varphi} \equiv \omega_{n m}$. Here $\Omega_{r}$ and $\Omega_{\varphi}$ are the two fundamental frequencies associated with the particle's radial and azimuthal motions, respectively. The integrals in Eqs. (14) and (15) thus reduce to summation over $n$. In particular,

$$
\phi_{l m}(t, r)=\sum_{n} R_{l m \omega_{n m}}(r) e^{-i \omega_{n m} t},
$$

where in principle the summation is over all integer values of $n$.

The physically-acceptable solutions of Eq. (16) are those satisfying the appropriate boundary conditions at both edges $r \rightarrow \infty$ and $r \rightarrow 2 M$, which correspond to pure outgoing waves at spatial infinity and pure incoming waves at the event horizon. The standard procedure for constructing the desired physical solution, for given $l m \omega$, begins with the construction of a basis of two independent homogeneous solutions, $R_{\operatorname{lm} \omega}^{+}$and $R_{\operatorname{lm} \omega}^{-}$. These two homogeneous solutions satisfy the required boundary conditions at, respectively, $r \rightarrow \infty$ and $r \rightarrow 2 M$ (note that there is no non-trivial homogeneous solution which satisfies the required boundary conditions at both edges). One then utilizes the standard Wronskian-based formula for generating inhomogeneous solutions to second-order linear differential equations. Transforming the integration variable from $r_{*}$ to $r$ using $d r / d r_{*}=f(r)$, and recalling the bounded support of $Z_{l m \omega}(r)$, this formula takes the form

$$
\begin{aligned}
R_{l m \omega}(r) & =R_{l m \omega}^{+}(r) \int_{r_{\min }}^{r} \frac{R_{l m \omega}^{-}\left(r^{\prime}\right) Z_{l m \omega}\left(r^{\prime}\right)}{W f\left(r^{\prime}\right)} d r^{\prime}+R_{l m \omega}^{-}(r) \int_{r}^{r_{\max }} \frac{R_{l m \omega}^{+}\left(r^{\prime}\right) Z_{l m \omega}\left(r^{\prime}\right)}{W f\left(r^{\prime}\right)} d r^{\prime} \\
& \equiv R_{l m \omega}^{\mathrm{inh}}(r),
\end{aligned}
$$

where

$$
W \equiv R_{l m \omega}^{-}\left(d R_{l m \omega}^{+} / d r_{*}\right)-R_{l m \omega}^{+}\left(d R_{l m \omega}^{-} / d r_{*}\right)=\mathrm{const}
$$

is the Wronskian. In the regions $r \leq r_{\min }$ and $r \geq r_{\max }$ this formula reduces to the homogeneous solutions

$$
R_{l m \omega}(r)= \begin{cases}C_{l m \omega}^{-} R_{l m \omega}^{-}(r) \equiv \tilde{R}_{l m \omega}^{-}(r), & r \leq r_{\min }, \\ C_{l m \omega}^{+} R_{l m \omega}^{+}(r) \equiv \tilde{R}_{l m \omega}^{+}(r), & r \geq r_{\max },\end{cases}
$$

where the coefficients $C_{l m \omega}^{-}$and $C_{l m \omega}^{+}$are given by

$$
C_{l m \omega}^{ \pm}=W^{-1} \int_{r_{\min }}^{r_{\max }} \frac{R_{l m \omega}^{\mp}(r) Z_{l m \omega}(r)}{f(r)} d r
$$


We conclude this section by explicitly writing the frequency-domain expressions for the three key functions $\phi_{l m}^{i}$, in the particle's neighborhood:

$$
\begin{gathered}
\phi_{l m}(t, r)=\sum_{n} R_{l m \omega_{n m}}^{\mathrm{inh}}(r) e^{-i \omega_{n m} t}, \\
\phi_{l m, r}(t, r)=\sum_{n} \frac{d}{d r} R_{l m \omega_{n m}}^{\mathrm{inh}}(r) e^{-i \omega_{n m} t}, \\
\phi_{l m, t}(t, r)=-i \sum_{n} \omega_{n m} R_{l m \omega_{n m}}^{\mathrm{inh}}(r) e^{-i \omega_{n m} t} .
\end{gathered}
$$

Since the particle resides in the range $r_{\min } \leq r \leq r_{\max }$, the radial functions $R_{l m \omega}^{\mathrm{inh}}(r)$ involved in these expressions are truly inhomogeneous [unlike the functions $\tilde{R}_{l m \omega}^{ \pm}(r)$ defined in Eq. [20), which are homogeneous]. [30]

\section{THE HIGH-FREQUENCY PROBLEM}

\section{A. Statement of the problem}

The functions $\phi_{l m}^{i}(t, r)$, whose one-sided values are required for the self-force calculation, are perfectly (one-sided) smooth, even at the coincidence limit $r \rightarrow r_{\mathrm{p}}(t)$. Owing to this smoothness, one may naturally expect that there ought to be a way to calculate the required one-sided quantities in the frequency domain without referring to large $\omega$ values. Such a method indeed exists, as we explain in the next section. However, with a straightforward application of the standard frequency-domain method, based on Eqs. (22)-(24), one finds that the Fourier series either fails to converge to the correct values (for $\phi_{l m, r}$ and $\phi_{l m, t}$ ) or converges very slowly (for $\phi_{l m}$ ) as $r \rightarrow r_{\mathrm{p}}(t)$.

To demonstrate this convergence problem we consider first the Fourier sum (23) for $\phi_{l m, r}$, which is required for calculating $F_{r}^{\text {self }}$. [Essentially the same argument applies to Eq. (24) for $\phi_{l m, t}$.] Suppose that we attempt to evaluate $\phi_{l m, r}$ at a point $x=x_{f}$ on the worldline, with coordinates $t=t_{f}$ and $r=r_{f} \equiv r_{\mathrm{p}}\left(t_{f}\right)$. The values of the radial functions $d R_{l m \omega}^{\mathrm{inh}} / d r$ at $r=r_{f}$ are just the Fourier components of the function

$$
\phi_{l m, r}^{f}(t) \equiv \phi_{l m, r}\left(r=r_{f}, t\right) .
$$

Since $\phi_{l m, r}$ is discontinuous at the worldline, $\phi_{l m, r}^{f}(t)$ is discontinuous at $t=t_{f}$ [as well as at any other $t$ value for which $r_{\mathrm{p}}(t)=r_{f}$ ]. We therefore encounter here the Gibbs Phenomenon [21]: If a function $F(t)$ is discontinuous, its Fourier sum will fail to converge to the correct value at the discontinuity. (Away from the discontinuity the Fourier sum will converge, but rather slowly and only conditionally: The $n$-th order term will behave as $1 / n$.)

Consider next the convergence properties of the Fourier sum for $\phi_{l m}$ in Eq. (22). Defining $\phi_{l m}^{f}(t) \equiv \phi_{l m}\left(r=r_{f}, t\right)$, we observe that $\phi_{l m}^{f}(t)$ is continuous, yet its derivative is discontinuous at $t=t_{f}$. Standard Fourier theory [20] then has it that the Fourier sum will indeed converge (to the correct value) at $r \rightarrow r_{\mathrm{p}}(t)$, but this convergence will be rather slow: The $n$-th term of the Fourier series is expected to behave as $1 / n^{2}$.

\section{B. Numerical illustration: Scalar-field monopole}

It is instructive to illustrate the above problem with an explicit calculation. For this, we consider the example of the monopole mode $l=m=0$. The spectrum in this case becomes simply $w=n \Omega_{r}$ (with integer $n$ ), and the Fourier sum (17) takes the form

$$
\phi(r, t)=\sum_{n=-\infty}^{\infty} R_{n}^{\mathrm{inh}}(r) e^{-i n \Omega_{r} t} .
$$

We hereafter use the notation $\phi_{l=m=0} \equiv \phi, R_{l=m=0, \omega=n \Omega_{r}} \equiv R_{n}$, etc. to represent the various monopole quantities. For a given orbit, the inhomogeneous $n$-mode radial functions $R_{n}^{\text {inh }}(r)$ can be computed numerically based on Eq. (18). The relevant numerical procedure is rather standard, and we relegate its description to App. C. In the following we present sample results and discuss their significance. 

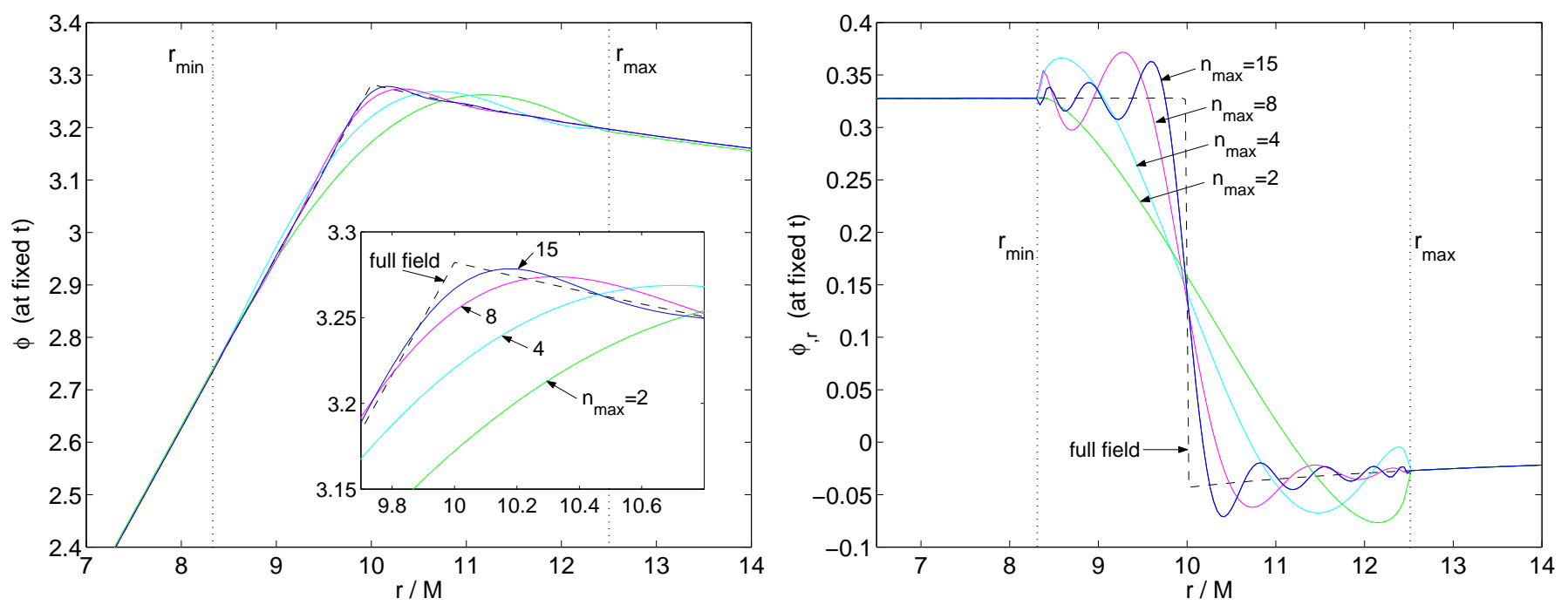

FIG. 1: Construction of the scalar-field monopole and its $r$ derivative as a sum over inhomogeneous frequency modes in the standard approach. The numerical solutions shown here correspond to an eccentric geodesic orbit around a Schwarzschild black hole, with semi-latus rectum $p=10$ and eccentricity $e=0.2$ (see App. A). The "periastron" and "apastron" for this orbit are at $r_{\min }=(25 / 3) M \cong 8.333 M$ and $r_{\max }=12.5 \mathrm{M}$, respectively. We used numerical integration to calculate the inhomogeneous radial functions $R_{n}^{\text {inh }}(r)$ through Eq. (C4), and then obtained the partial sums $\phi\left(t, r ; n_{\max }\right)$ and $\phi_{, r}\left(t, r ; n_{\max }\right)$ as defined in Eqs. (27) and (28). Plotted here (solid lines) are the partial sums (per unit scalar charge) for $n_{\max }=2,4,8,15$, as functions of $r$ at a fixed time $t$ when the particle is at $r=10 M$ (this corresponds to radial phase $\chi=\pi / 2$; see App. A]. The left panel displays the scalar field itself; the right panel shows its $r$ derivative. For comparison, we also display (dashed line) the full scalar monopole solution, which we obtained directly using numerical evolution in the time domain (for our purpose, this latter solution can be taken as an accurate benchmark). It is evident that the $n$-mode sum converges quickly to the correct value in the regions $r<r_{\min }$ and $r>r_{\max }$, but the convergence deteriorates inside the domain $r_{\min }<r<r_{\max }$, where "Gibbs waves" set in. The partial sum over frequency modes is smooth at the particle, and hence, strictly speaking, cannot recover the true jump discontinuity in the field derivative there. Finding a way around this technical problem is the main goal of this work.

Figures 1 and 2 display numerical solutions for the sample orbital parameters $r_{\max }=12.5 M$ and $r_{\min }=(25 / 3) M \cong$ $8.333 M$. (This corresponds to "semi-latus rectum" $p=10 M$ and "eccentricity" $e=0.2$, both quantities defined in App. A.) In Fig. 1 1 we plot the (real-valued) partial sums

$$
\phi\left(r, t ; n_{\max }\right) \equiv \sum_{n=-n_{\max }}^{n_{\max }} R_{n}^{\mathrm{inh}}(r) e^{-i n \Omega_{n} t}
$$

and

$$
\phi_{, r}\left(r, t ; n_{\max }\right) \equiv \sum_{n=-n_{\max }}^{n_{\max }} \frac{d}{d r} R_{n}^{\mathrm{inh}}(r) e^{-i n \Omega_{n} t}
$$

as functions of $r$ at the fixed time $t$ when the particle is located at $r=10 M$, for a sample of $n_{\max }$ values. For comparison, we also plot the full monopole solution (and its $r$ derivative), which we obtain using a time-domain numerical evolution code similar to that developed by Haas in Ref. [16]. Evidently (and as expected), the convergence of the $n$-mode sum for both $\phi$ and $\phi_{, r}$ seems very fast at $r<r_{\min }$ and $r>r_{\max }$, but deteriorates significantly in the domain $r_{\min }<r<r_{\max }$, where "Gibbs waves" dominate the behavior. Figure2 2 illustrates the convergence properties of the partial sums $\phi\left(n_{\max }\right)$ and $\phi_{, r}\left(n_{\max }\right)$ at the very location of the particle (on the same time slice as in Fig. 1). The data on the left panel suggest that the partial sum for the field $\phi$ converges at the particle as $\sim 1 / n_{\max }$-in accordance with theoretical expectation [20]. The results shown in the right panel of Fig. 2 demonstrate that the partial sum for the derivative $\phi_{, r}$ fails to converge to the correct (one-sided) values. They also (loosely) suggest that this partial sum in fact converges to the two-side average value of $\phi_{, r}$ at the particle. This, indeed, would again accord with theoretical prediction [20].

\section{Practical implications of the high-frequency problem}

The above numerical example serves to illustrate the following: From a practical point of view, it would seem very difficult to extract the correct values of the key functions $\phi_{l m}^{i}$ at the particle's location, based plainly on a naive 

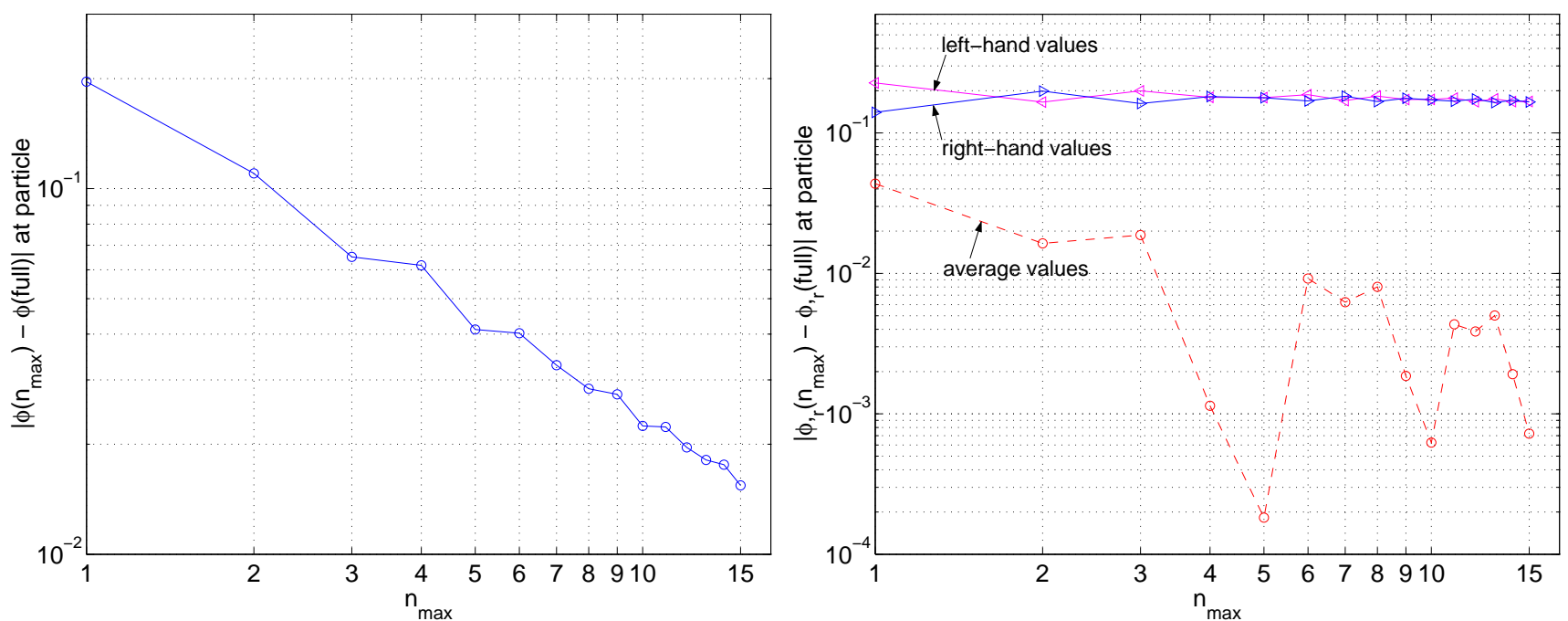

FIG. 2: Convergence of the Fourier $n$-mode sum at the location of the particle (illustrated here based on the numerical data of Fig. 1). Left panel: The deviation (per unit scalar charge) of the partial sum $\phi\left(n_{\max }\right)$ from the full field $\phi$ at the location of the particle, as a function of $n_{\max }$. The $n$-mode partial sum appears to converge to the correct value approximately as $1 / n_{\max }$ (note the log-log scale), as expected on theoretical grounds. Right panel: The deviation (per unit scalar charge) of $\phi_{, r}\left(n_{\max }\right)$ from the full-field derivative $\phi_{, r}$, again evaluated at the particle's location. Since the true $\phi_{, r}\left[\right.$ unlike $\left.\phi_{, r}\left(n_{\max }\right)\right]$ has two different one-sided values at $r=r_{\mathrm{p}}$, so does the deviation. The two solid lines represent these two one-sided values of the deviation. (These two values actually have opposite signs for each $n_{\max }$, which is obscured here since only the absolute value of the deviation is shown.) From the essentially-horizontal shape of the two solid lines it is evident that the $n$-mode sum for $\phi_{, r}$ does not converge to the correct one-sided values. As an aside, we also plot here (dashed line) the difference between $\phi_{, r}\left(n_{\max }\right)$ and the two-side average value of the full derivative $\phi_{, r}$ (per unit scalar charge, at the particle's location). The graph loosely suggests (referring to its upper envelop and ignoring the seemingly oscillatory deep structure) that $\phi_{, r}\left(n_{\max }\right)$ converges, albeit very slowly, to the average value of $\phi_{, r}$ at the discontinuity. That, indeed, would again be consistent with theoretical prediction.

summation over frequency modes as in Eqs. (22)-(24). The partial Fourier sum for the field $\phi_{l m}$ itself would converge very slowly $($ as $1 / n)$ and its evaluation would hence be computationally expensive. Worse, the partial sums for the derivatives $\phi_{l m, r}$ and $\phi_{l m, t}$ would simply fail to yield the desired one-sided values at the particle, even if one could sum over infinitely many modes.

Having stated the above, we should also point out that Eqs. (23) and (24) should not be deemed entirely useless for the purpose of calculating $\phi_{l m, r}$ and $\phi_{l m, t}$ at the particle. In principle, one could pick a point close to $r=r_{\mathrm{p}}(t)$, yet not quite at $r_{\mathrm{p}}(t)$, and calculate $\phi_{l m, r}$ (say) there. The Fourier sum will converge at this point, although rather slowly. One could then pick a series of $r$-values which approach $r_{\mathrm{p}}(t)$ (say, from the ' + ' side), calculate $\phi_{l m, r}$ at each of these points, and then evaluate the desired sided-limit of $\phi_{l m, r}$ through extrapolation. This procedure, however, is cumbersome and is hardly likely to be computationally tractable.

An alternative implementation strategy could make use of the fact that the sums in Eqs. (23) and (24) actually converge to the two-side averages of $\phi_{l m, r}$ and $\phi_{l m, t}$ at the particle (recall the discussion relating to Fig. 22). These average values (along with $\phi_{l m}$ itself) could be used to construct the average force modes $\bar{F}_{l \mu} \equiv\left(F_{l \mu}^{+}+F_{l \mu}^{-}\right) / 2$, which could then be directly implemented in a "two-side averaged" version of the mode sum formula: $F_{\mu}^{\text {self }}=$ $\sum_{l=0}^{\infty}\left[\bar{F}_{l \mu}\left(x_{f}\right)-B_{\mu}\right]$. Although this method is likely to be by far more efficient than the extrapolation method mentioned earlier, it would still present a computational challenge, as this method, too, involves the evaluation of slowly-converging Fourier sums.

\section{METHOD OF EXTENDED HOMOGENEOUS SOLUTIONS}

\section{A. Formulation of method}

In this section we describe our alternative method for frequency-domain construction of the key quantities $\phi_{l m}^{i}$ required for calculation of the self force. This method, to which we refer as the method of extended homogeneous solutions, completely avoids the high-frequency problem described above and ensures exponentially-fast convergence of the Fourier series. 
We begin by extending the definition of the homogeneous functions $\tilde{R}_{l m \omega}^{ \pm}(r)$ in Eq. (20) to the entire domain $r>2 M$ :

$$
\tilde{R}_{l m \omega}^{ \pm}(r) \equiv C_{l m \omega}^{ \pm} R_{l m \omega}^{ \pm}(r), \quad r>2 M
$$

We then define the two time-domain extended homogeneous solutions $\tilde{\phi}_{l m}^{+}$and $\tilde{\phi}_{l m}^{-}$to be the outcome of replacing $R_{l m \omega}^{\mathrm{inh}}$ in Eq. (22) by the homogeneous solutions $\tilde{R}_{l m \omega}^{+}$or $\tilde{R}_{l m \omega}^{-}$, respectively:

$$
\tilde{\phi}_{l m}^{ \pm}(t, r) \equiv \sum_{n} \tilde{R}_{l m \omega_{n m}}^{ \pm}(r) e^{-i \omega_{n m} t}
$$

We emphasize that each of the fields $\tilde{\phi}_{l m}^{+}$and $\tilde{\phi}_{l m}^{-}$is defined in the entire domain $2 M<r<\infty$.

The convergence properties of the sum in Eq. (30) are dictated by the large- $|n|$ asymptotic behavior of the coefficients $\tilde{R}_{l m \omega_{n m}}^{ \pm}(r)$. This high-frequency asymptotic behavior can be examined using a WKB-type analysis, which we carry out in App. D This analysis shows that, at least within the leading-order WKB approximation, the terms on the righthand side of Eq. (30) decay (at least) exponentially in $|n|$. This exponential decay is uniform in $t$ and $r$, throughout $r>2 M$. Also, one naturally expects that the contribution from higher-order terms in the large- $\omega$ WKB expansion will converge even faster than the leading-order contribution, and hence will not affect this uniform exponential decay.

The exponential convergence of the sum (30) is extremely convenient for numerical applications, as illustrated in the next subsection. But it also has important mathematical consequences. Since the homogeneous radial functions $\tilde{R}_{l m \omega}^{ \pm}(r)$ are analytic, the uniform exponential decay of the individual terms in the above sum implies that the overall sum - namely the extended homogeneous solution $\tilde{\phi}_{l m}^{ \pm}(t, r)$-is an analytic function of $r$ and $t$ throughout $r>2 M$.

We now argue that on each side of the curve $r=r_{\mathrm{p}}(t)$ the actual time-domain function $\phi_{l m}(t, r)$ coincides with one of these extended homogeneous solutions, namely

$$
\phi_{l m}(t, r)= \begin{cases}\tilde{\phi}_{l m}^{+}(t, r), & r \geq r_{\mathrm{p}}(t), \\ \tilde{\phi}_{l m}^{-}(t, r), & r \leq r_{\mathrm{p}}(t) .\end{cases}
$$

For concreteness, let us present our argument explicitly referring to the first of these equalities: (i) In the domain $r>r_{\max }$ this equality obviously holds because $R_{l m \omega}^{i n h}$ and $\tilde{R}_{l m \omega}^{+}$coincide in that domain. (ii) As was already mentioned in Sec. III, we assume that the function $\phi_{l m}(t, r)$ is analytic throughout the range $r>r_{\mathrm{p}}(t)$ [as well as in the other range, $2 M<r<r_{\mathrm{p}}(t)$ ], because the alternative option appears unreasonable. (iii) As was just discussed above, the high-frequency analysis in App. D strongly suggests that the extended homogeneous functions $\tilde{\phi}_{l m}^{+}(t, r)$ are analytic throughout $r>2 M$. (iv) Since both functions $\phi_{l m}$ and $\tilde{\phi}_{l m}^{+}$are analytic throughout the domain $r>r_{\mathrm{p}}(t)$ [from (ii) and (iii)], and coincide at $r>r_{\max }$ [from (i)], they must coincide throughout $r>r_{\mathrm{p}}(t)$. (v) By continuity of both functions $\phi_{l m}$ and $\tilde{\phi}_{l m}^{+}$, they coincide at $r=r_{\mathrm{p}}(t)$ as well. Obviously, the same line of argument applies to the second of the equalities (31) as well.

In the rest of this subsection we describe the utility of the extended homogeneous fields defined above in calculations of the self-force via the mode-sum method. Recall from Eqs. (11) -(13) that this method requires as input (either of) the one-sided limits of $\phi_{l m}^{i}$ at the particle, which we now denote

$$
\phi_{l m}^{i \pm}\left(x_{f}\right) \equiv \lim _{x \rightarrow x_{f \pm}} \phi_{l m}^{i}
$$

In terms of $\phi_{l m}^{i \pm}$, the various components of the quantities $F_{l \mu}^{ \pm}\left(x_{f}\right)$ [as defined through Eqs. (11) and (12) and used in the mode-sum formula (13)] are expressed directly as

$$
\left\{F_{l t}^{ \pm}, F_{l r}^{ \pm}, F_{l \varphi}^{ \pm}\right\}=\frac{q}{r_{f}} \sum_{m=-l}^{l}\left\{\phi_{l m, t}^{ \pm}, \phi_{l m, r}^{ \pm}-\phi_{l m}^{ \pm} / r_{f}, i m \phi_{l m}^{ \pm}\right\} Y_{l m}\left(\pi / 2, \varphi_{f}\right)
$$

(along with $F_{l \theta}^{ \pm}=0$ ).

For concreteness, let us focus first on one of the quantities $\phi_{l m}^{i \pm}$, say $\phi_{l m}^{+}$. By definition, the limit $x \rightarrow x_{f+}$ in Eq. (32) only samples the range $r>r_{\mathrm{p}}(t)$. Using Eqs. (31) and (30) we may re-express $\phi_{l m}^{+}$as

$$
\phi_{l m}^{+}\left(x_{f}\right)=\lim _{x \rightarrow x_{f+}} \sum_{n} \tilde{R}_{l m \omega_{n m}}^{+}(r) e^{-i \omega_{n m} t} .
$$


Since the sum over $n$ here converges uniformly, we may interchange the limit and summation. However, the functions $\tilde{R}_{l m \omega_{n m}}^{+}$and $e^{-i \omega_{n m} t}$ are analytic, so we can now omit the limit $x \rightarrow x_{f+}$ and instead simply evaluate these functions at $x=x_{f}$. The final outcome from these manipulations is stated in (the ' + ' case of) Eq. (35) below.

The above treatment is equally applicable to $\phi_{l m}^{-}$, and we obtain a similar formula for constructing $\phi_{l m}^{-}\left(x_{f}\right)$ out of the extended homogeneous modes $\tilde{R}_{l m \omega_{n m}}^{-}(r)$ [the '-' case of Eq. (35)]. Moreover, the same treatment also applies to $\phi_{l m, r}^{ \pm}$and $\phi_{l m, t}^{ \pm}$. The six key quantities $\phi_{l m}^{i \pm}$ can all be constructed from the extended homogeneous radial functions (and their derivatives) in the form

$$
\begin{gathered}
\phi_{l m}^{ \pm}\left(x_{f}\right)=\sum_{n} \tilde{R}_{l m \omega_{n m}}^{ \pm}\left(r_{f}\right) e^{-i \omega_{n m} t_{f}}, \\
\phi_{l m, r}^{ \pm}\left(x_{f}\right)=\sum_{n} \frac{d}{d r} \tilde{R}_{l m \omega_{n m}}^{ \pm}\left(r_{f}\right) e^{-i \omega_{n m} t_{f}}, \\
\phi_{l m, t}^{ \pm}\left(x_{f}\right)=-i \sum_{n} \omega_{n m} \tilde{R}_{l m \omega_{n m}}^{ \pm}\left(r_{f}\right) e^{-i \omega_{n m} t_{f}} .
\end{gathered}
$$

Equations (29) and (35)-(37), combined with Eqs. (33) and (13), constitute our new method of calculating the self force in the frequency domain. The high-frequency problem is entirely circumvented in this method, as the Fourier sum converges exponentially-fast for all functions $\phi_{l m}^{i \pm}$.

\section{B. Numerical illustration: Scalar-field monopole revisited}

Let us revisit the calculation of the scalar-field monopole - this time using the method of extended homogeneous solutions. The homogeneous basis solutions $R_{n}^{ \pm}(r)$ are constructed numerically in just the same manner as in the standard approach (see App. B). Then, however, instead of calculating the actual inhomogeneous modes $R_{n}^{\mathrm{inh}}(r)$ as in Sec. III. we construct the extended homogeneous solutions $\tilde{R}_{n}^{ \pm}(r)$ as they are defined in Eq. (29), with the coefficients $C_{l=m=0, \omega=n \Omega_{r}}^{ \pm} \equiv C_{n}^{ \pm}$calculated through Eq. (C7) of App. C] The time-domain extended fields and their $r$ derivatives are then approximated by the (real-valued) partial sums

$$
\begin{gathered}
\tilde{\phi}^{ \pm}\left(r, t ; n_{\max }\right)=\sum_{n=-n_{\max }}^{n_{\max }} \tilde{R}_{n}^{ \pm}(r) e^{-i n \Omega_{n} t}, \\
\tilde{\phi}_{, r}^{ \pm}\left(r, t ; n_{\max }\right)=\sum_{n=-n_{\max }}^{n_{\max }} \frac{d}{d r} \tilde{R}_{n}^{ \pm}(r) e^{-i n \Omega_{n} t},
\end{gathered}
$$

with sufficiently large $n_{\max }$.

We point out the following matters relating to the implementation of Eqs. (38) and (39): (i) In the new approach, the computation of $\tilde{\phi}$ (or $\tilde{\phi}_{, r}$ ) for all $r$ and $t$ involves the (numerical) evaluation of only two integrals - the ones in Eq. (C7) of App. C] In contrast, the standard approach requires the evaluation of two integrals - the ones in Eq. (C4) separately for each value of $r$ between $r_{\min }$ and $r_{\max }$. (ii) The full scalar monopole is continuous at $r=r_{\mathrm{p}}(t)$; however, the contributions to the extended functions $\tilde{\phi}^{+}$and $\tilde{\phi}^{-}$from each individual $n$ mode do not match continuously along this curve. Consequently, for any finite $n_{\max }$, the partial sums for these extended functions do not match at $r=r_{\mathrm{p}}(t)$. The amplitude of this mismatch is expected to decrease rapidly with growing $n_{\max }$, as the results below indeed demonstrate.

Figures 3 [5 display numerical solutions obtained based on Eqs. (38) and (39). Our goal here is to assess the performance of the new method against the standard method, and to this end we have chosen for our numerical experiment the same orbital parameters as in Figs. 1 1 and 2 of Sec. III. For clarity, we only show the ' + ' and ' - ' fields in their respective relevant domains, i.e., $r \geq r_{\mathrm{p}}(t)$ for the former and $r \leq r_{\mathrm{p}}(t)$ for the latter.

Our numerical illustration serves to demonstrate the following: (i) The sum over ' + ' and ' - ' extended $n$-modes converges quickly to the correct, full solution everywhere in the respective domains $r \geq r_{\mathrm{p}}(t)$ and $r \leq r_{\mathrm{p}}(t)$. (ii) In particular, the mismatch between the values of the ' + ' and '-' partial sums at the particle's location quickly converges to zero with growing $n_{\max }$. (iii) The convergence of the extended $n$-mode sum is exponential everywhere even in the region $r_{\min } \leq r \leq r_{\max }$; in particular, it is exponential at the very location of the particle. This applies to both the field and its derivatives. (iv) The new scheme completely circumvents the Gibbs effect which disrupts the convergence of the inhomogeneous $n$-modes in the standard approach. 

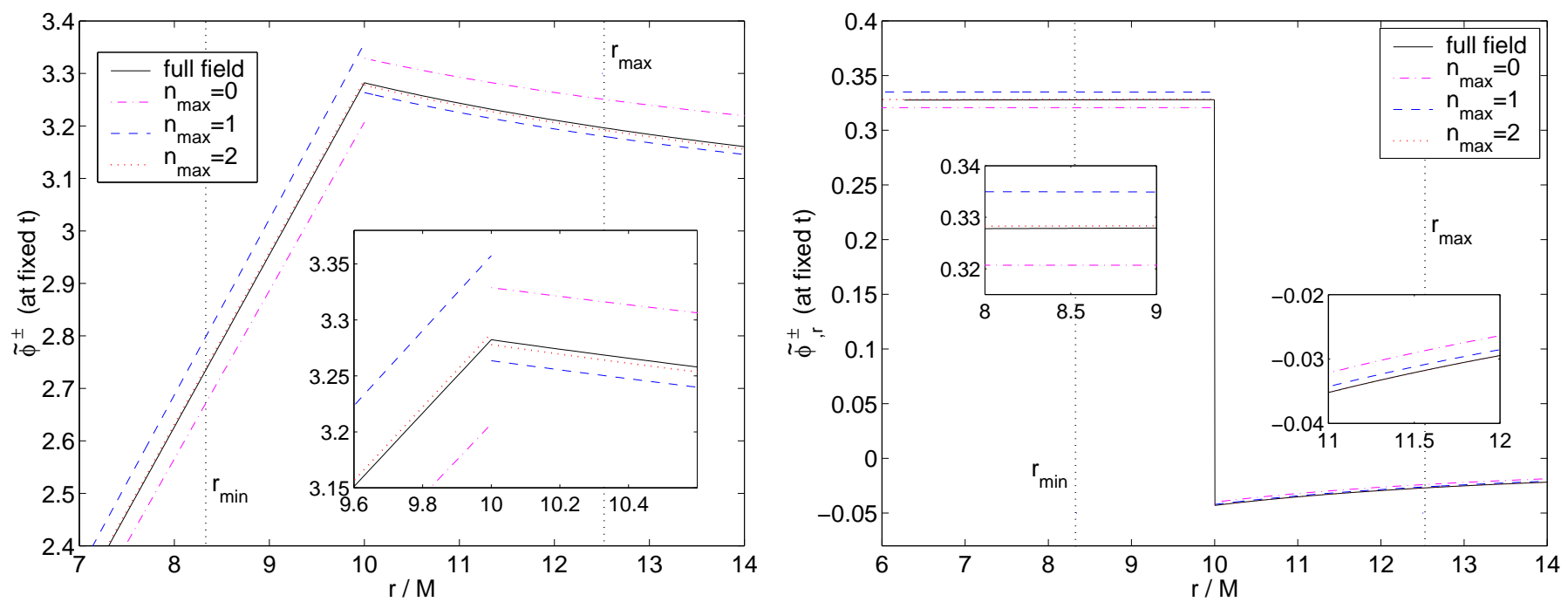

FIG. 3: (To be compared with Fig. 10) Construction of the scalar-field monopole (left panel) and its $r$ derivative (right panel) using "extended homogeneous solutions". The orbital parameters are chosen here as in Fig. 1 i.e., $p=10$ and $e=0.2$, and we again present the various fields as functions of $r$ at a fixed time $t$ when the particle is at $r=10 M$. Vertical dotted lines mark the particle's periastron and apastron radii, $r_{\min }=(25 / 3) M$ and $r_{\max }=12.5 M$, respectively. The solid line represents the full scalar monopole solution, obtained using numerical evolution in the time domain. The broken lines represent partial sums over extended homogeneous $n$-modes, calculated numerically based on Eqs. (38) and (39). For clarity, in both panels we plot the ' + ' and ' - ' partial sums only in their relevant domains, $r \geq 10 M$ and $r \leq 10 M$, respectively. We show here the partial sums for $n_{\text {max }}=0,1,2$ only-the partial sums $\tilde{\phi}^{ \pm}\left(n_{\max }=3\right)$ are already indistinguishable from the full solution at the scale of this plot (but see Fig. 团below). The individual $n$-modes of the extended fields $\tilde{\phi}^{+}$ and $\tilde{\phi}^{-}$do not match continuously at the location of the particle, but their sum seems to converge quickly, everywhere, to the true solution (which is continuous). Similar fast convergence is manifest also for the derivative $\phi_{, r}$. "Gibbs waves", which disrupt the convergence of the actual inhomogeneous $n$-modes, are altogether avoided within the new scheme.
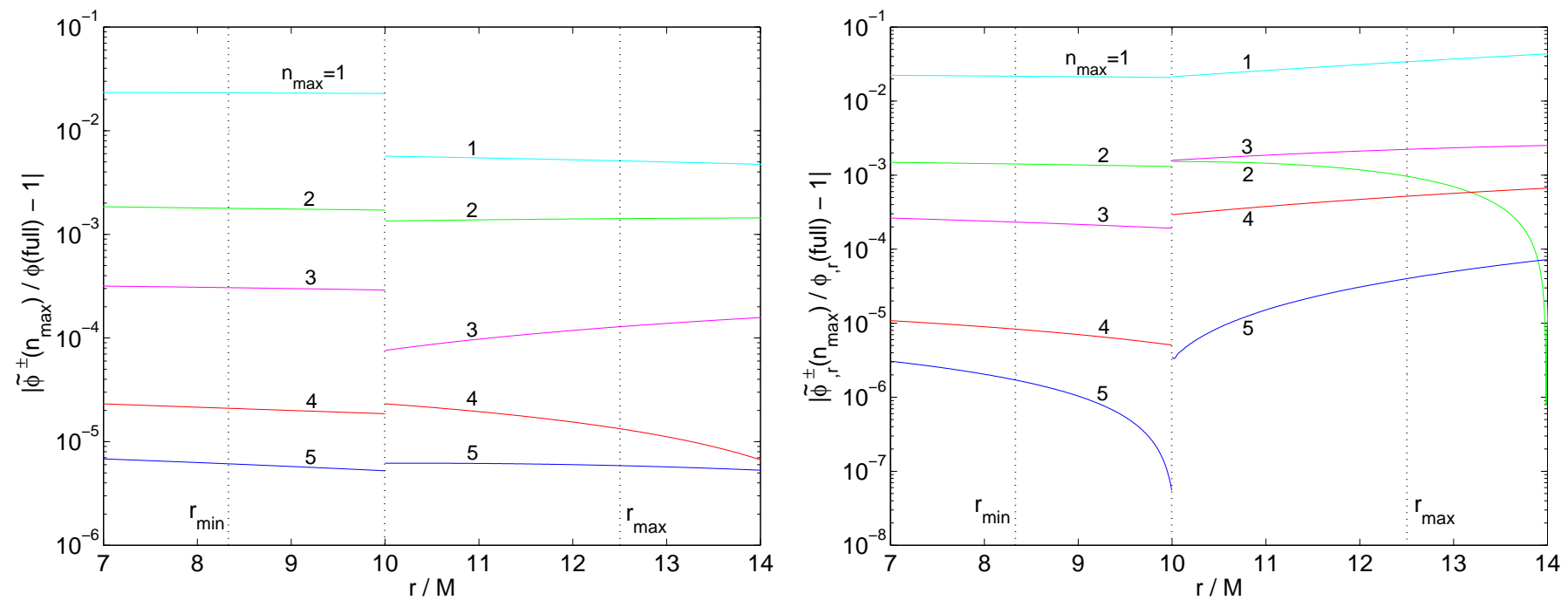

FIG. 4: Convergence of the extended $n$-mode sum for the fields $\tilde{\phi}^{ \pm}$(left panel) and their derivatives $\tilde{\phi}_{, r}^{ \pm}$(right panel). For the same case shown in Fig. 3 we plot here the fractional differences between the partial sums and the full field (or the full-field derivative), for $n_{\max }=1-5$. The various graphs are labeled by their corresponding $n_{\max }$ values. The middle vertical dotted line marks the particle's momentary radius at $r=10 \mathrm{M}$. Once again, we display the ' + ' and ' - ' values only in their respective relevant domains $r \geq 10 M$ or $r \leq 10 M$. Note the exponential scale of the y-axis. (The seemingly odd behavior of the data for $n_{\max }=2$ and $n_{\max }=5$ in the right panel is simply due to a change-of-sign which the corresponding fractional differences happen to experience around $r=14 M$ and $r=10 M$, respectively. The tiny wiggly feature, barely visible near $r=10 M$ for $n_{\max }=5$, is due to the numerical error in the time-domain data, which for $\phi_{, r}$ is estimated at $\sim 10^{-6}$ in fractional terms.) The exponentially-fast convergence of the extended $n$-mode sum even at $r_{\min } \leq r \leq r_{\max }$ - and particularly at the very location of the particle-is evident from these plots. 

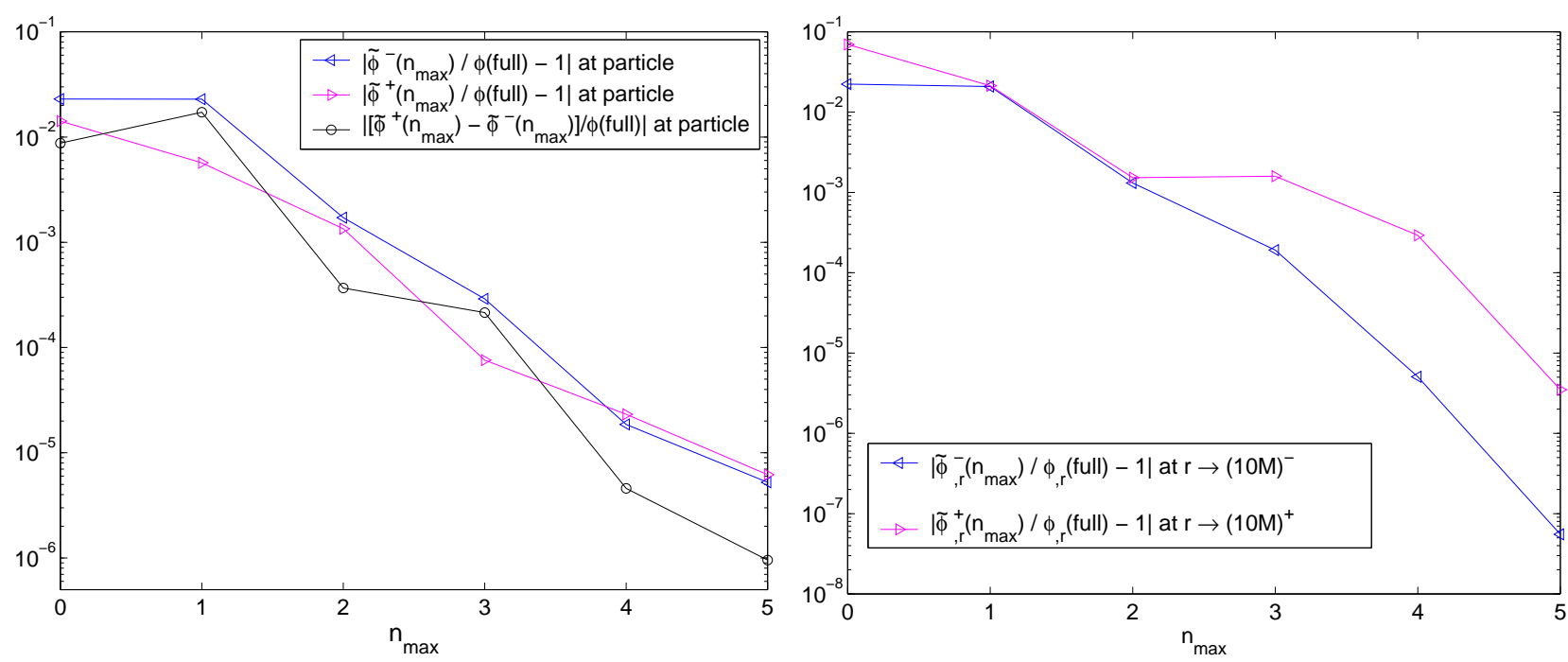

FIG. 5: Convergence of the extended $n$-mode sum at the particle's location. The left and right panels display the values at the particle's location $(r=10 M)$ of the fractional differences shown in the left and right panels of Fig. 4 correspondingly. The left panel demonstrates the exponential convergence of the extended $n$-mode sum to the correct value of the field at the particle. In particular, the mismatch between the partial sums for $\tilde{\phi}^{+}$and $\tilde{\phi}^{-}$at the particle appears to vanish exponentially with increasing $n_{\max }$. As we suggest in Sec. $\nabla$ one can in fact make a good use of this mismatch in numerical calculations, by recording its residual value and using it to assess the truncation error of the partial $n$-mode sum. Comparing with Figs. 1 and 2 (left panels), it is striking that, in the example considered here, summing up to only $n=2$ with the new method achieves a better accuracy in the local field than summing over as many as 16 modes using the standard approach. The right panel demonstrates the fast convergence of the derivatives $\tilde{\phi}_{, r}^{+}$and $\tilde{\phi}_{, r}^{-}$in the new approach. An exponential convergence, expected form theory, is loosely suggested from the data shown.

\section{DISCUSSION}

The basic relation underpinning our new method is expressed in Eq. (31), which describes the construction of the $l m$-multipole of the scalar field from extended homogeneous solutions. Eqsuation (29) and (35)-(37), in conjunction with Eqs. (33) and (13), constitute our new prescription for constructing the self force in the frequency domain. The following list highlights the advantages of this new formulation.

- In the standard frequency-domain scheme, the Fourier sum over $\omega$-modes suffers from Gibbs-type irregularities near the particle's location. In particular, the Fourier sum fails to correctly recover the derivatives of the field's multipoles at the particle (which are essential input in self-force calculations). In the new method one constructs the local field's multipoles as Fourier sums of globally homogeneous $\omega$-modes. These sums converge uniformly, circumventing the above complication. This is the essential and most crucial merit of the new method. [Note also that the uniform convergence (which also applies to the derivatives of the extended homogeneous mode functions) allows one to obtain the derivatives of the field's multipoles using a term-by-term differentiation of the individual Fourier components. This again leads to Eqs. (36) and (37) above.]

- The sum over the homogeneous $\omega$ modes converges exponentially everywhere, and even at the very location of the particle. This is extremely convenient from a practical point of view. It should be noted that, within our new scheme, it becomes "as easy" to obtain the local $l$-mode field near the particle as it is to obtain the same field in the far-zone - in sharp contrast with the situation in both the standard frequency-domain method and the time-domain method.

- In the standard scheme, each of the inhomogeneous $\omega$-modes is computed via Eq. (18). In practice, this involves the (numerical) evaluation of two integrals for each value of $r$ between $r_{\min }$ and $r_{\max }$. In the new scheme, the extended homogeneous $\omega$-modes are obtained via Eq. (29), which requires the same two integrals (21) for all values of $r$. Thus, remarkably, the new scheme does not only perform better mathematically - it is also much simpler to implement.

- Finally, within the new scheme one is offered a convenient handle with which to monitor and control the large- $\omega$ truncation error (i.e., the error caused by omission of the terms $|n|>n_{\max }$ ): The residual amount by which the partial sum $\sum_{n=-n_{\max }}^{n_{\max }}\left(\right.$ for any of the quantities $\phi_{l m}^{i}$ ) fails to be consistent with the appropriate jump condition 
at $r=r_{\mathrm{p}}(t)$ is a faithful measure of the truncation error. One can therefore conveniently keep the latter below a set level by setting a threshold on the amount of residual inconsistency.

What is the application scope of the new method? In this paper we introduced the method as applied specifically for scalar-field perturbations on the Schwarzschild background. However, the basic idea is equally applicable to a wide range of other problems. It is clear from our discussion that the problematics of reconstructing the local multipole field as a sum over frequency modes has little bearing on the precise form of the underlying field equations. Rather, it is a feature of the spherical-harmonic decomposition: When we consider an individual $l m$ mode, we effectively convert the physical problem of a point particle moving in an eccentric orbit into a problem of a source "shell" (which expands and contracts over time); the perturbation field is not smooth across the shell, which gives rise to Gibbs-type complications when we attempt to express it as a sum over frequency modes. The same problem would occur in essentially any perturbation treatment in Schwarzschild which incorporates a spherical harmonic decomposition, such as the Teukolsky formalism (for EM and gravitational perturbations), the standard Regge-Wheeler/Zerilli/Moncrief formalism of gravitational perturbations, or the more direct Lorenz-gauge formulation [23]. The method we propose here as a cure for the problem is directly applicable for any of these treatments. A forthcoming paper [19] will report on the calculation of the monopole and dipole modes of the Lorenz-gauge metric perturbation (for eccentric orbits in Schwarzschild), facilitated by the method of extended homogeneous solutions. This calculation is now being incorporated in a code which computes the total gravitational self-force for generic orbits in Schwarzschild [24].

To what extent is our new method relevant for Kerr perturbations? Here the situation is more subtle. The original mode-sum scheme for the self force [10] (which sets the main context for the current work) incorporates a decomposition in spherical harmonics even in the Kerr spacetime. This is, of course, technically possible (see the footnoted remark at the Introduction), although the resulting field equations then couple between different $l$-modes. Regardless of the latter fact, each of the $l m$-modes in this decomposition would again be sourced by an expanding/contracting thin shell, the non-smoothness of the perturbation across this shell would give rise to the Gibbs phenomenon, and our method would provide an efficient cure.

The situation is different if one tackles the Kerr problem by means of the more natural decomposition in spheroidal harmonics, which decouples the field equation in the frequency domain. Since the spheroidal-harmonic functions depend on the frequency, one no longer has a strict notion of a time-domain ' $l m$ mode' in this case. One may (somewhat artificially) define the "spheroidal-harmonic $l^{\prime} m$-mode" $\Phi_{l^{\prime} m}(t, r, \theta, \varphi)$ by summing over all $\omega$ for given spheroidal-harmonic numbers $l^{\prime}, m$. However, in this case the effective geometric picture of a thin source shell would no longer apply: In the procedure of Fourier decomposition of the original point source (to obtain the source's $l^{\prime} m \omega$ modes) followed by a Fourier summation over $\omega$, the extra dependence of the spheroidal harmonics on $\omega$ will cause the reconstructed source's $l^{\prime} m$ mode function to deviate from a $\delta$-function in $t$ (for given $r, \theta, \varphi$ ). Correspondingly, at a given $t$ the source's $l^{\prime} m$ mode function will most likely represent a "smeared" shell.

Based on the above discussion one might conclude that the high-frequency problem would not occur in the first place if spheroidal harmonics were used (as in this case there would be no $\delta$-type shell). We believe, however, that this may represent a false logic. The viability of the mode-sum approach relies crucially on the fact that the individual mode contributions $F_{l \mu}^{ \pm}$in Eq. (13) are well-defined quantities. This fact is a direct consequence of the perfect one-sided smoothness of the mode functions $\phi_{l m}(t, r)$ even at the limit $r \rightarrow r_{\mathrm{p}}(t)$. This smoothness, in turn, stems from the fact that for each spherical-harmonic $l, m$ the source term is confined to a $\delta$-function over a shell-and this $\delta$-function is distributed over the shell in a perfectly smooth manner. Note also that the functions $\phi_{l m}(t, r)$ are $h o m o g e n e o u s$ time-domain solutions on both sides of this shell-even at the immediate particle's neighborhood. In spheroidalharmonic decomposition this situation is changed, and the spheroidal-harmonic mode function $\Phi_{l^{\prime} m}(t, r, \theta, \varphi)$ defined above will no longer be a homogeneous solution in the very neighborhood of the particle. It is conceivable that in the immediate particle's neighborhood the smeared source shell will be dominated by large- $\omega$ modes. [The sphericalharmonics decomposition is protected against this potential problem thanks to the combination of (i) the perfect off-shell homogeneity, and (ii) the independence of the $l, m$ harmonic - and hence of the $\delta$-function distribution over the spherical shell - on $\omega$.$] Thus, in a spheroidal-harmonic decomposition, the high-frequency problem may take a$ much more severe form: It may endanger the very existence of $F_{l^{\prime} \mu}^{ \pm}$(namely the spheroidal-harmonics analogs of $F_{l \mu}^{ \pm}$) as regular quantities, which would in turn render the (spheroidal-harmonics analog of the) mode-sum formula (13) meaningless.

The morphology of the spheroidal-harmonics smeared source shell still needs be investigated, and especially its structure near the point charge. The mode contributions $F_{l^{\prime} \mu}^{ \pm}$may turn out to be well-defined after all, but this is far from obvious. In any case, a spheroidal-harmonics-based variant of the mode-sum method for Kerr has not been developed yet to the best of our knowledge. The existing Kerr mode-sum variant [10] incorporates the sphericalharmonics decomposition, and as such it exhibits the same high-frequency problem as in the Schwarzschild case. The method of extended homogeneous solutions then elegantly resolves this problem in the Kerr case as well. 


\section{ACKNOWLEDGEMENTS}

LB and NS acknowledge support from PPARC/STFC through grant number PP/D001110/1.

\section{APPENDIX A: ECCENTRIC GEODESICS IN SCHWARZSCHILD}

This appendix reviews the standard description of eccentric geodesics in Schwarzschild spacetime, and provides the necessary formulas for all orbital quantities (frequency, four-velocity, etc.) needed for the numerical computations in Secs. III and IV]

As in the main text, we consider a pointlike test particle in a bound equatorial geodesic orbit around a Schwarzschild black hole with mass parameter $M$. The radial location of the particle is bounded in the range $r_{\min } \leq r \leq r_{\max }$, for some $r_{\max }>r_{\min }>4 M$. Such geodesics constitute a two-parameter family. Each geodesic is uniquely characterized, for example, by the two "turning point" values $r_{\min }$ and $r_{\max }$. An alternative parameterization (originally due to Darwin [25]) employs the "semi-latus rectum", $p$, and "eccentricity", $e$, both analogous to their counterparts from Keplerian celestial mechanics. The parameter pairs $(p, e)$ and $\left(r_{\min }, r_{\max }\right)$ are related through

$$
p=\frac{2 r_{\max } r_{\min }}{r_{\max }+r_{\min }}, \quad e=\frac{r_{\max }-r_{\min }}{r_{\max }+r_{\min }},
$$

or, inverting,

$$
r_{\max }=\frac{p}{1-e}, \quad r_{\min }=\frac{p}{1+e} .
$$

With the parameterization $(p, e)$, the orbital radius is given by

$$
r_{\mathrm{p}}(\chi)=\frac{p}{1+e \cos \chi},
$$

where $\chi$ is a monotonically-increasing parameter ("radial phase") along the worldline. This parameter is related to the Schwarzschild time $t_{\mathrm{p}}$ along the worldline through

$$
\frac{d \chi}{d t_{\mathrm{p}}}=\frac{(p-2 M-2 M e \cos \chi)(1+e \cos \chi)^{2}}{p^{2}}\left(\frac{p / M-6-2 e \cos \chi}{(p / M-2)^{2}-4 e^{2}}\right)^{1 / 2},
$$

with the constant of integration fixed such that $\chi=0$ at some "periastron" passage $\left(r_{\mathrm{p}}=r_{\min }\right) \cdot \chi$ is related to the proper time along the eccentric geodesic through

$$
\frac{d \chi}{d \tau}=\frac{(1+e \cos \chi)^{2}}{M(p / M)^{3 / 2}}\left(\frac{p / M-6-2 e \cos \chi}{p / M-3-e^{2}}\right)^{1 / 2} .
$$

The radius $r_{\mathrm{p}}$ is manifestly periodic in $\chi$, with $t$-period

$$
T_{r} \equiv \int_{0}^{2 \pi}\left(d \chi / d t_{\mathrm{p}}\right)^{-1} d \chi
$$

and radial frequency $\Omega_{r} \equiv 2 \pi / T_{r}$.

For our numerical implementation of Eqs. (C4) and (C7) (in App. [C), we start by choosing $r_{\min }$ and $r_{\max }$ and then use Eq. (A1) to determine $p$ and $e$ [or, alternatively, we pick $p$ and $e$, then use Eq. (A2) to determine $r_{\min }$ and $r_{\max }$ ]. We then solve for $t_{\mathrm{p}}(\chi)$ for $0 \leq \chi \leq \pi$ by integrating the inverse of Eq. (A4) numerically [taking $t_{\mathrm{p}}(0)=0$ ]. We hence obtain the radial period $T_{r}=2 t_{\mathrm{p}}(\chi=\pi)$ and the radial frequency $\Omega_{r}$. Using Eq. (A33) to express $\chi$ in terms of $r$ along the orbit (again for $0 \leq \chi \leq \pi$ ), we then obtain $t_{\mathrm{p}}(\chi(r)) \equiv t_{\mathrm{p}}(r)$, and, inverting in the range $0 \leq t_{\mathrm{p}} \leq T_{r} / 2$, also $r_{\mathrm{p}}(t)$. Finally, $u^{t}$ is obtained as a function of $t$ by writing $u^{t}=\left(d t_{\mathrm{p}} / d \chi\right)(d \chi / d \tau)$, substituting from Eqs. (A4) and (A5), and then using Eq. (A3) to express $\chi$ in terms of $r_{\mathrm{p}}(t)$. This procedure yields all necessary orbital parameters and functions for our numerical examples.

Since the numerical illustrations of this work focus on the monopole mode, which is axially-symmetric, they do not require an explicit computation of the azimuthal frequency $\Omega_{\varphi}$. For completeness, though, we mention that this frequency is defined, in an orbit-average manner, by

$$
\Omega_{\varphi} \equiv \frac{1}{T_{r}} \int_{0}^{T_{r}}\left(d \varphi_{\mathrm{p}} / d t\right) d t
$$


The "local" frequency, which depends on $r_{\mathrm{p}}$, is given in terms of the $r$-phase $\chi$ as

$$
\frac{d \varphi_{\mathrm{p}}}{d t}=\frac{(p / M-2-2 e \cos \chi)(1+e \cos \chi)^{2}}{M(p / M)^{3 / 2}\left[(p / M-2)^{2}-4 e^{2}\right]^{1 / 2}} .
$$

The frequency $\Omega_{\varphi}$ can be computed by changing the integration variable in Eq. (A7) to $\chi$, using Eq. (A4).

\section{APPENDIX B: NUMERICAL CONSTRUCTION OF THE HOMOGENEOUS SOLUTIONS $R_{n}^{ \pm}(r)$}

We describe here the numerical construction of the frequency-domain homogeneous basis $\left(R_{n}^{+}, R_{n}^{-}\right)$in the example of the scalar-field monopole. For $l=0$, the homogeneous part of the $n$-mode radial ODE (16) takes the form

$$
\frac{d^{2} R_{n}^{ \pm}}{d r_{*}^{2}}+\left[n^{2} \Omega_{r}^{2}-2 M f(r) r^{-3}\right] R_{n}^{ \pm}=0 .
$$

For each $n, R_{n}^{+}$and $R_{n}^{-}$are independent solutions of this equation, satisfying physical boundary conditions at $r \rightarrow \infty$ and $r \rightarrow 2 M$, respectively. These homogeneous solutions are needed both in constructing the 'true' inhomogeneous $n$-mode solution $R_{n}^{\text {inh }}$ [via Eq. (C4) in App. C] and in constructing the extended homogeneous solutions $\tilde{R}_{n}^{ \pm}$within our new method [via Eqs. (29) and (C7)].

Consider first the static mode, $n=0$, which can be solved for analytically: We have, simply, $R_{0}^{-}=r$ and $R_{0}^{+}=r \ln f$, which constitutes a unique basis (up to multiplicative constants) with the property that $R_{0}^{-}$is regular at the event horizon and $R_{0}^{+}$is regular at $r \rightarrow \infty$. [To see this, recall that the static mode of the actual, full scalar field is $\Phi \propto R_{0}^{ \pm} / r$, with corresponding "internal" solution $\Phi^{-} \propto \operatorname{const}(\neq 0)$ and "external" solution $\Phi^{+} \propto \ln f$. The former is regular at the horizon but fails to vanish at infinity, while the latter falls off as $1 / r$ at infinity but diverges at the horizon.]

For each mode $n \neq 0$ we solve Eq. (B1) numerically with suitable boundary conditions, as we now describe. Our numerical domain is a one-dimensional array representing physical radii in the range $r_{\text {in }} \leq r \leq r_{\text {out }}$. The boundaries are taken to lie in the asymptotic vacuum domains: $r_{\text {in }} /(2 M)-1 \ll 1$ (the event horizon) and $r_{\text {out }} \gg M$ (spatial infinity). In practice, it proved sufficient for our purpose to set $r_{\text {in }}=2.001 M$ and $r_{\text {out }}=1000 M$.

Consider first $R_{n}^{-}$. As an inner boundary condition for this function we use the ansatz

$$
R_{n}^{-}(r)=e^{-i n \Omega_{r} r_{*}} \sum_{k=0}^{k_{\text {max }}^{-}} a_{n k}^{-}(r-2 M)^{k} \quad\left(\text { at } r=r_{\text {in }}\right),
$$

where $a_{k}^{-}$are coefficients to be determined below, and $k_{\max }^{-}$is taken large enough to guarantee that truncation error is kept below a prescribed threshold (in practice, $k_{\max }^{-}=10$ proved sufficient for the values of $e, p, n$ considered in this work). The oscillatory factor in Eq. (B2) is chosen such that the contribution from each $n$-mode to the full monopole field attains the asymptotic form $\propto \exp \left[-i n \Omega_{r}\left(t+r_{*}\right)\right]$ as $r \rightarrow 2 M$ [recall Eq. (26)]. This represents purely ingoing radiation, which is the correct physical condition at the event horizon. To determine the coefficients $a_{n k}^{-}$we substitute Eq. (B2) in the field equation (B1) and solve the resulting hierarchy of algebraic equations at each order in $r-2 M$. This yields the following recursion formula for the coefficients $a_{n k}^{-}$(with given $n$; we omit here the index $n$ for brevity):

$$
\begin{aligned}
a_{k>0}^{-}= & \frac{-1}{2 M^{2} k\left(k-4 i M n \Omega_{r}\right)} \times\left\{\left[M(2 k-1)(k-2)-12 i M^{2} n \Omega_{r}(k-1)\right] a_{k-1}^{-}\right. \\
& \left.+\left[(k-3) / 2-6 i M n \Omega_{r}\right](k-2) a_{k-2}^{-}-i n \Omega_{r}(k-3) a_{k-3}^{-}\right\}
\end{aligned}
$$

with $a_{k<0}^{-}=0$. All coefficients $a_{k>0}^{-}$are constructed recursively given $a_{0}^{-}$, and are all proportional to $a_{0}^{-}$.

To solve Eq. (B1) for $R_{n}^{-}(r)$, we simply set $a_{0}^{-}=1$, impose the values of $R_{n}^{-}$and $d R_{n}^{-} / d r$ at $r_{\text {in }}$ using Eq. (B2), and integrate numerically forward from $r=r_{\text {in }}$ to $r=r_{\max }$ (the value of $R_{n}^{-}$at $r>r_{\text {max }}$ is not needed in our analysis).

Now consider $R_{n}^{+}$. As an outer boundary condition for this function we take

$$
R_{n}^{+}(r)=e^{i n \Omega_{r} r_{*}} \sum_{k=0}^{k_{\max }^{+}} a_{n k}^{+} r^{-k} \quad\left(\text { at } r=r_{\text {out }}\right),
$$

where $a_{n k}^{+}$are determined below, and $k_{\max }^{+}$is chosen, once again, such that truncation error is kept below a set threshold (here, too, $k_{\max }^{+}=10$ was sufficient in our analysis). With this condition, the contribution from each $n$ mode to the full monopole field has the asymptotic form $\propto \exp \left[-i n \Omega_{r}\left(t-r_{*}\right)\right]$ as $r \rightarrow \infty$, representing purely outgoing 
radiation - the correct physical condition at infinity. The expansion coefficients in Eq. (B4) are obtained recursively using

$$
a_{k>0}^{+}=\frac{i}{2 n \Omega_{r} k}\left[-k(k-1) a_{k-1}^{+}+2 M(k-1)^{2} a_{k-2}^{+}\right],
$$

with $a_{k<0}^{+}=0$. (Recall $n=0$ is dealt with analytically, so one need not worry about the ill-definiteness of the recursion relation in this case.) All coefficients $a_{k>0}^{+}$are constructed recursively given $a_{0}^{+}$, and are all proportional to $a_{0}^{+}$.

To solve Eq. (B1) for $R_{n}^{+}$, we set $a_{0}^{+}=1$, impose the values of $R_{n}^{+}$and $d R_{n}^{+} / d r$ at $r_{\text {out }}$ using Eq. (B4), and integrate numerically backward from $r=r_{\text {out }}$ to $r=r_{\min }$ (the value of $R_{n}^{+}$at $r<r_{\min }$ is not needed in our analysis).

\section{APPENDIX C: CONSTRUCTION OF THE INHOMOGENEOUS SOLUTIONS $R_{n}^{\text {inh }}$}

This Appendix details the computation of the inhomogeneous radial functions $R_{n}^{\text {inh }}(r)$ for our numerical illustration in Sec. IIIB.

For $l=0$, the functions $R_{n}^{\text {inh }}(r)$ satisfy the ODE

$$
\frac{d^{2} R_{n}^{\mathrm{inh}}}{d r_{*}^{2}}+\left[n^{2} \Omega_{r}^{2}-2 M f(r) r^{-3}\right] R_{n}^{\mathrm{inh}}=Z_{n}
$$

where the source term is obtained by taking the inverse-Fourier transform in (the discrete, $l=0$ version of) Eq. (15):

$$
\begin{aligned}
Z_{n}(r) & =T_{r}^{-1} \int_{0}^{T_{r}}\left[-4 \pi r f(r) \hat{\rho}_{l=m=0}(t, r)\right] e^{i n \Omega_{r} t} d t \\
& =-q(4 \pi)^{1 / 2} T_{r}^{-1} \int_{0}^{T_{r}} f(r)\left(r u^{t}\right)^{-1} \delta\left[r-r_{\mathrm{p}}(t)\right] e^{i n \Omega_{r} t} d t
\end{aligned}
$$

Here $T_{r}$ is the radial period [see Eq. (A6)], and in the second equality we have substituted for $\hat{\rho}$ from Eq. (7), setting $m=0$ and $\hat{c}_{l=m=0}=(4 \pi)^{-1 / 2}$. Note in Eq. (C2) that $Z_{n}=0$ for $r<r_{\min }$ or $r>r_{\max }$, and that for any $r_{\min }<r<r_{\max }$ the integrand is supported only at two points within the integration domain-the two times $t$ for which $r_{\mathrm{p}}(t)=r$. Changing the integration variable from $t$ to $r_{\mathrm{p}}$ one thus obtains

$$
Z_{n}(r)=-\frac{2 q(4 \pi)^{1 / 2} f(r)}{T_{r} r\left|u^{r}(r)\right|} \cos \left[n \Omega_{n} t_{\mathrm{p}}(r)\right] \times \Theta\left(r-r_{\min }\right) \times \Theta\left(r_{\max }-r\right),
$$

where $\Theta$ is the standard unit step function, $u^{r}=d r_{\mathrm{p}} / d \tau$ is the $r$ component of the particle's four-velocity, and $t_{\mathrm{p}}(r)$ is the result of inverting $r=r_{\mathrm{p}}(t)$ in the domain $0 \leq t \leq T_{r} / 2$, assuming $r_{\mathrm{p}}(0)=r_{\min }$ [note $r_{\mathrm{p}}(t)$ is single-valued in this restricted domain].

In the standard approach, the physical solution to Eq. (C1) is obtained through the formula (18), replacing $R_{l m \omega} \rightarrow$ $R_{n}^{\text {inh }}, R_{l m \omega}^{ \pm} \rightarrow R_{n}^{ \pm}$, and $Z_{l m \omega} \rightarrow Z_{n}$. Here $R_{n}^{+}$and $R_{n}^{-}$are two independent solutions to the homogeneous part of Eq. (C1), satisfying physical boundary conditions at $r \rightarrow \infty$ and $r=2 M$, respectively. It is convenient to change the integration variable in Eq. (18) from $r$ to $t_{\mathrm{p}}(r)$ [taking $t_{\mathrm{p}}\left(r_{\min }\right)=0$ ], which avoids the singularity in $Z_{n}(r)$ at $r=r_{\max }, r_{\min }$. Substituting for $Z_{n}$ from Eq. (C3), and using $\left|u^{r}\right|=u^{r}=u^{t}\left(d r_{\mathrm{p}} / d t\right)$ for $0 \leq t \leq T_{r} / 2$, Eq. (18) becomes

$$
\begin{aligned}
R_{n}^{\mathrm{inh}}(r)=-2 q(4 \pi)^{1 / 2} T_{r}^{-1} W^{-1} \times & {\left[R_{n}^{+}(r) \int_{0}^{\hat{t}_{\mathrm{p}}(r)} \frac{R_{n}^{-}\left(r_{\mathrm{p}}(t)\right)}{r_{\mathrm{p}}(t) u^{t}\left(r_{\mathrm{p}}(t)\right)} \cos \left(n \Omega_{r} t\right) d t\right.} \\
& \left.+R_{n}^{-}(r) \int_{\hat{t}_{\mathrm{p}}(r)}^{T_{r} / 2} \frac{R_{n}^{+}\left(r_{\mathrm{p}}(t)\right)}{r_{\mathrm{p}}(t) u^{t}\left(r_{\mathrm{p}}(t)\right)} \cos \left(n \Omega_{r} t\right) d t\right]
\end{aligned}
$$

where we have introduced

$$
\hat{t}_{\mathrm{p}}(r)= \begin{cases}0, & r \leq r_{\min } \\ t_{\mathrm{p}}(r), & r_{\min } \leq r \leq r_{\max } \\ T_{r} / 2, & r \geq r_{\max }\end{cases}
$$


The solution $R_{n}^{\mathrm{inh}}$ in Eq. (C4) is manifestly an analytic function of $r$ everywhere, except at $r=r_{\min }, r_{\max }$. Note, recalling Eq. (20), that outside the domain $r_{\min }<r<r_{\max }$ Eq. (C4) reduces to the homogeneous solutions

$$
R_{n}^{\mathrm{inh}}(r)=\left\{\begin{array}{l}
C_{n}^{-} R_{l m \omega}^{-}(r), r \leq r_{\min }, \\
C_{n}^{+} R_{l m \omega}^{+}(r), r \geq r_{\max },
\end{array}\right.
$$

where the coefficients $C_{n}^{ \pm}$are given by

$$
C_{n}^{ \pm}=-2 q(4 \pi)^{1 / 2} T_{r}^{-1} W^{-1} \int_{0}^{T_{r} / 2} \frac{R_{n}^{\mp}\left(r_{\mathrm{p}}(t)\right)}{r_{\mathrm{p}}(t) u^{t}\left(r_{\mathrm{p}}(t)\right)} \cos \left(n \Omega_{r} t\right) d t .
$$

Equation (C4) can be implemented numerically to obtain $R_{n}^{\text {inh }}(r)$, in the following manner: We specify the physical orbit by picking the values of $r_{\min }$ and $r_{\max }$, and then use the relations given in App. $\AA$ to obtain (numerically) the values of $\Omega_{r}$ and $T_{r}$ and the functions $r_{\mathrm{p}}(t), t_{\mathrm{p}}(r)$ and $u^{t}\left(r_{\mathrm{p}}(t)\right)$ for the specified orbit. We next construct the homogeneous basis $R_{n}^{ \pm}(r)$ by numerically integrating the homogeneous part of Eq. (C1) with suitable boundary conditions. This procedure is described in App. B. Once the solutions $R_{n}^{ \pm}(r)$ are at hand, the (constant) value of the Wronskian is obtained using Eq. (19). Finally, for each given $n$, we calculate the integrals in Eq. (C4) numerically, and construct the solution $R_{n}^{\text {inh }}(r)$.

\section{APPENDIX D: HIGH-FREQUENCY ANALYSIS}

In this appendix we analyze the convergence and analyticity of the Fourier sum involved in the construction of the key functions $\phi_{l m}^{(i)}$ in the "extended homogeneous solutions" approach-i.e., the sum over $n$ on the right-hand side of Eq. (30). Both convergence and analyticity are crucial for the definiteness and validity of our proposed approach. By analyzing the behavior of the mode sum in the high-frequency limit, we provide here a strong indication this sum converges (at least) exponentially in the entire domain $r>2 M$, and is therefore also analytic in this entire domain.

\section{WKB approximation for the large- $\omega$ homogeneous radial functions}

The extended frequency-domain radial functions are give by

$$
\tilde{R}_{l m \omega}^{ \pm}(r)=C_{l m \omega}^{ \pm} R_{l m \omega}^{ \pm}(r) \quad(r>2 M),
$$

where the (r-independent) coefficients $C_{l m \omega}^{ \pm}$are given in Eq. (21), and $\left\{R_{l m \omega}^{+}(r), R_{l m \omega}^{-}(r)\right\}$ is a pair of independent solutions to the homogeneous equation

$$
\frac{d^{2} R_{l m \omega}}{d r_{*}^{2}}+\left[\omega^{2}-V_{l}(r)\right] R_{l m \omega}=0,
$$

satisfying suitable boundary conditions at $r \rightarrow \infty$ and $r \rightarrow 2 M$, respectively. In what follows we shall explore the asymptotic behavior of the quantities $\tilde{R}_{l m \omega}^{ \pm}$at large $\omega$, and consequently evaluate the large- $\omega$ contribution to the extended time-domain functions $\tilde{\phi}_{l m}^{ \pm}(t, r)$ in Eq. (35).

Since the potential $V_{l}(r)$ is bounded (for a given $l$ ), in the large- $\omega$ limit the term in square brackets in Eq. (D2) is dominated by $\omega^{2}$. Using the WKB approximation, we can then write

$$
R_{l m \omega}^{ \pm} \cong\left[1-V_{l}(r) / \omega^{2}\right]^{-1 / 4} \exp \left( \pm i \int \sqrt{\omega^{2}-V_{l}(r)} d r_{*}\right) .
$$

The square-root term may be expanded for large $\omega$ as

$$
\sqrt{\omega^{2}-V_{l}(r)} \cong \omega-V_{l}(r) /(2 \omega) .
$$

We shall only consider here the leading-order solution at large $\omega$, so we ignore the term $V_{l}(r) /(2 \omega)$ in this expression, as well as the $\propto \omega^{-2}$ term in the square brackets in Eq. (D3). We obtain

$$
R_{l m \omega}^{ \pm} \cong e^{ \pm i \omega r_{*}} \text {. }
$$

From the form of the ODE (D2) it is clear that the Wronskian $W$ is constant, and for the specific pair $R_{l m \omega}^{ \pm}$in Eq. (D5) it takes the value

$$
W=2 i \omega .
$$




\section{The source term $Z_{l m \omega}$ and its spectrum}

To calculate the coefficients $C_{l m \omega}^{ \pm}$we first need to analyze the source term $Z_{l m \omega}(r)$ and determine its discrete spectrum. Inverting the Fourier transform in Eq. (15), we have

$$
Z_{l m \omega}(r)=f^{[1]}(r) \int_{0}^{T_{r}} \hat{\rho}_{l m}(t, r) e^{i \omega t} d t
$$

where $T_{r}$ denotes the $t$-period of the radial motion (see App. A). Throughout this appendix, $f^{[k]}(k=1,2,3, \ldots)$ denote certain functions of $r$, independent of $\omega$ (or $n$ ), which are analytic throughout $r>2 M$ (but whose precise form would not interest us otherwise).

Our first goal is to obtain the $\omega$ spectrum of $\hat{\rho}_{l m}$. For an eccentric geodesic the azimuthal motion may be expressed as

$$
\varphi_{\mathrm{p}}(t)=\Omega_{\varphi} t+\Delta \varphi(t)
$$

where $\Omega_{\varphi}$ is the $t$-averaged angular frequency $d \varphi / d t$ (see App. $\mathrm{A}$ ), and $\Delta \varphi(t)$ is a certain analytic function of $t$, which is periodic with periodicity $T_{r}$. [Analyticity is directly inherited from that of $\varphi_{\mathrm{p}}(t)$; The $T_{r}$-periodicity results from the fact that for a given orbit $d \Delta \varphi / d t$ is a function of $r$ only.] Substituting this in Eq. (7) we get

$$
\hat{\rho}_{l m}(t, r)=\hat{c}_{l m} q\left(r^{2} u^{t}\right)^{-1} \beta_{m}(t) e^{-i m \Omega_{\varphi} t} \delta\left[r-r_{\mathrm{p}}(t)\right]
$$

where $\beta_{m}(t) \equiv e^{-i m \Delta \varphi(t)}$ is an analytic, $T_{r}$-periodic, function of $t$. We rewrite this as

$$
\begin{aligned}
\hat{\rho}_{l m}(t, r) & =\left(q f_{l m}^{[2]}(r) \beta_{m}(t) \delta\left[r-r_{\mathrm{p}}(t)\right]\right) e^{-i m \Omega_{\varphi} t} \\
& \equiv S_{l m}(t, r) e^{-i m \Omega_{\varphi} t} .
\end{aligned}
$$

The spectrum of $\hat{\rho}_{l m}$ is the same as that of $S_{l m}$, with all frequencies simply shifted by $m \Omega_{\varphi}$. Now, the dependence of $S_{l m}$ on $t$ is only through the $T_{r}$-periodic functions $r_{\mathrm{p}}(t)$ and $\beta_{m}(t)$. Therefore we may write

$$
S_{l m}(t, r)=\sum_{n} S_{l m n}(r) e^{-i n \Omega_{r} t}
$$

and correspondingly

$$
\hat{\rho}_{l m}(t, r)=\sum_{n} S_{l m n}(r) e^{-i\left(m \Omega_{\varphi}+n \Omega_{r}\right) t}
$$

where $\Omega_{r}=2 \pi / T_{r}$ is the fundamental radial frequency. Thus, the spectrum of $\hat{\rho}_{l m}(t, r)$ is the discrete set of frequencies

$$
\omega=m \Omega_{\varphi}+n \Omega_{r} \equiv \omega_{n m}
$$

Finally, we calculate the coefficients $Z_{l m n}(r) \equiv Z_{l m \omega_{n m}}(r)$ using Eq. (D7). Pulling the factor $f^{[1]}(r)$ into the integral and using Eqs. (D10) and (D13), we get

$$
Z_{l m n}(r)=q \int_{0}^{T_{r}} f_{l m}^{[3]}(r) \beta_{m}(t) \delta\left[r-r_{\mathrm{p}}(t)\right] e^{i n \Omega_{r} t} d t .
$$

\section{Calculating the coefficients $C_{l m \omega}^{ \pm}$}

We turn now to calculate the coefficients $C_{l m n}^{ \pm} \equiv C_{l m \omega_{n m}}^{ \pm}$. These are given by

$$
C_{l m n}^{ \pm}=W^{-1} \int_{r_{\min }}^{r_{\max }} \frac{R_{l m n}^{\mp}(r) Z_{l m n}(r)}{f(r)} d r
$$

where $R_{l m n}^{\mp} \equiv R_{l m \omega_{n m}}^{\mp}$. Substituting from Eq. (可14) for $Z_{l m n}$ and absorbing the factor $1 / f(r)$ in $f_{l m}^{[3]}$ (to form another analytic function, $f_{l m}^{[4]}$ ) we get

$$
C_{l m n}^{ \pm}=q W^{-1} \int_{r_{\min }}^{r_{\max }} \int_{0}^{T_{r}} f_{l m}^{[4]}(r) R_{l m n}^{\mp}(r) \beta_{m}(t) \delta\left[r-r_{\mathrm{p}}(t)\right] e^{i n \Omega_{r} t} d t d r
$$


The two integrals in the last equation are allowed to be interchanged (as one may verify, for example, by explicitly carrying out first the $t$-integral and only then the $r$-integral). One obtains

$$
C_{l m n}^{ \pm}=q W^{-1} \int_{0}^{T_{r}} f_{l m}^{[4]}\left[r_{\mathrm{p}}(t)\right] R_{l m n}^{\mp}\left[r_{\mathrm{p}}(t)\right] \beta_{m}(t) e^{i n \Omega_{r} t} d t
$$

Next we substitute for $W$ from Eq. (D6), and also for $R_{l m n}^{\mp}$ using the large- $\omega$ asymptotic form given in Eq. (D5). Denoting $r_{*}^{\mathrm{P}}(t) \equiv r_{*}\left[r=r_{\mathrm{p}}(t)\right]$, we thus obtain

$$
\begin{aligned}
C_{l m n}^{ \pm} & \cong(-i / 2) q \omega_{n m}^{-1} \int_{0}^{T_{r}} f_{l m}^{[4]}\left[r_{\mathrm{p}}(t)\right] \beta_{m}(t) e^{\mp i \omega_{n m} r_{*}^{\mathrm{p}}(t)} e^{i n \Omega_{r} t} d t \\
& =(-i / 2) q \omega_{n m}^{-1} \int_{0}^{T_{r}} f_{l m}^{[4]}\left[r_{\mathrm{p}}(t)\right] \beta_{m}(t) e^{\mp i m \Omega_{\varphi} r_{*}^{\mathrm{p}}(t)} e^{i n \Omega_{r}\left[t \mp r_{*}^{\mathrm{p}}(t)\right]} d t .
\end{aligned}
$$

The analytic factor $e^{\mp i m \Omega_{\varphi} r_{*}^{\mathrm{P}}(t)}$ (as well as $-i / 2$ ) may be absorbed in $f_{l m}^{[4]}$-which will in turn become a new analytic function, denoted $f_{l m}^{[5] \mp}$. Defining

$$
t_{ \pm} \equiv t \pm r_{*}
$$

(namely the two Eddington-Finkelstein null coordinates), the last equation becomes

$$
C_{l m n}^{\mp} \cong q \omega_{n m}^{-1} \int_{0}^{T_{r}} f_{l m}^{[5] \pm}\left[r_{\mathrm{p}}(t)\right] \beta_{m}(t) e^{i n \Omega_{r} t_{ \pm}^{\mathrm{p}}(t)} d t
$$

where $t_{ \pm}^{\mathrm{p}}(t)$ is $t_{ \pm}\left(r=r_{\mathrm{p}}(t)\right) \equiv t \pm r_{*}^{\mathrm{p}}(t)$.

In the next step we wish to transform the integration variable from $t$ to $t_{ \pm}^{\mathrm{p}}(t)$. To this end we briefly discuss the properties of this transformation (and its inverse), particularly in terms of analyticity and periodicity. Obviously $t_{ \pm}^{\mathrm{p}}(t)$ is analytic. Also, since the orbit is timelike, $t_{ \pm}^{\mathrm{p}}(t)$ is monotonically increasing, and $d t_{ \pm}^{\mathrm{p}} / d t$ nowhere vanishes. This implies that the inverse function $t\left(t_{ \pm}^{\mathrm{p}}\right)$ is well-defined and analytic. Note also that all functions of $r_{\mathrm{p}}$ are periodic in $t_{ \pm}^{\mathrm{p}}$, with the same period $T_{r}$. The same applies to $\beta_{m}(t)$. Therefore Eq. (D19) may be expressed as [31]

$$
C_{l m n}^{\mp} \cong q \omega_{n m}^{-1} \int_{0}^{T_{r}} f_{l m}^{[5] \pm}\left[r_{\mathrm{p}}\left(t_{ \pm}^{\mathrm{p}}\right)\right] \beta_{m}\left[t\left(t_{ \pm}^{\mathrm{p}}\right)\right] \frac{d t}{d t_{ \pm}^{\mathrm{p}}} e^{i n \Omega_{r} t_{ \pm}^{\mathrm{p}}} d t_{ \pm}^{\mathrm{p}} .
$$

The last expression is nothing but $\left(q T_{r} \omega_{n m}^{-1}\right)$ times the $n$-th Fourier coefficient of the periodic function

$$
H_{l m n}^{ \pm}\left(t_{ \pm}^{\mathrm{p}}\right) \equiv f_{l m}^{[5] \pm}\left[r_{\mathrm{p}}\left(t_{ \pm}^{\mathrm{p}}\right)\right] \beta_{m}\left[t\left(t_{ \pm}^{\mathrm{p}}\right)\right] \frac{d t}{d t_{ \pm}^{\mathrm{p}}}
$$

Since all three factors on the right-hand side are analytic functions of $t_{ \pm}^{\mathrm{p}}$, so is $H_{l m n}^{ \pm}\left(t_{ \pm}^{\mathrm{p}}\right)$. Therefore its Fourier coefficients must decay (at least) exponentially with $|n|$, and the same applies to the coefficients $C_{l m n}^{\mp}$.

\section{Reconstructing the time-domain extended homogeneous solutions}

We turn now to explore the high- $\omega$ contribution to the time-domain extended homogeneous solutions

$$
\tilde{\phi}_{l m}^{ \pm}(t, r)=\sum_{n} \tilde{R}_{l m n}^{ \pm}(r) e^{-i \omega_{n m} t}=\sum_{n} C_{l m n}^{ \pm} R_{l m n}^{ \pm}(r) e^{-i \omega_{n m} t}
$$

Using the large- $\omega$ asymptotic expression (D5) one finds for the large- $\omega$ contribution

$$
\begin{aligned}
\tilde{\phi}_{l m}^{ \pm}(t, r) & \cong \sum_{n} C_{l m n}^{ \pm} e^{i \omega_{n m}\left(-t \pm r_{*}^{\mathrm{p}}\right)}=\sum_{n} C_{l m n}^{ \pm} e^{-i \omega_{n m} t_{\mp}} \\
& =e^{-i m \Omega_{\varphi} t_{\mp}} \sum_{n} C_{l m n}^{ \pm} e^{-i n \Omega_{r} t_{\mp}} .
\end{aligned}
$$

The sum in the last expression has the form of a Fourier series in time $t_{\mp}$, with coefficients $C_{l m n}^{ \pm}$which, as we already established, decay at least exponentially. Therefore, this sum converges to an analytic function of $t_{\mp}$. It then follows 
that the entire right-most expression in Eq. (D23) is analytic in $t_{\mp}$. Since $t_{\mp}$, in turn, is an analytic function of $r$ and $t$, then so must be this expression. We therefore conclude that, at least within the leading-order approximation, the large- $\omega$ contribution to $\tilde{\phi}_{l m}^{ \pm}(t, r)$ is analytic in both $r$ and $t$ throughout $r>2 M$. Note also that the sum over $n$ in Eq. (D23) is guaranteed to converge uniformly for all $t$ and $r>2 M$. The same applies to the corresponding Fourier sums for the $r$ and $t$ derivatives of $\tilde{\phi}_{l m}^{ \pm}$.

In the above discussion we considered only the leading-order term in the $1 / \omega$ WKB expansion. One naturally expects that the contribution from higher-order terms in this expansion will converge even faster, and hence will not interfere with the analyticity of $\tilde{\phi}_{l m}^{ \pm}(t, r)$. Also, such higher-order contributions are not expected to affect the uniform convergence of the sum over extended $n$ modes.

We regard the results of the above leading-order calculation as a strong indication that the extended time-domain solutions, as they are defined in Eq. (30), are indeed analytic everywhere outside the black hole. This is further supported by the numerical results presented in Sec. IVB.

[1] Y. Mino, M. Sasaki, and T. Tanaka, Phys. Rev. D 55, 3457 (1997)

[2] T. C. Quinn and R. M. Wald, Phys. Rev. D 56, 3381 (1997).

[3] S. E. Gralla and R. M. Wald, arXiv:0806.3293 [gr-qc].

[4] E. Poisson, Living Rev. Relativity, 7, 6 (2004) arXiv:gr-qc/0306052.

[5] Gravitational radiation from binary black holes: Advances in the perturbative approach, ed. C. O. Lousto [Class. Quantum Grav. 22, S543 (2005)].

[6] T. C. Quinn, Phys. Rev. D 62, 064029 (2000).

[7] B. S. DeWitt and R. W. Brehme, Ann. of Phys. 9, 220 (1960).

[8] L. Barack and A. Ori, Phys. Rev. D 61, 061502 (2000) arXiv:gr-qc/9912010.

[9] L. Barack, Y. Mino, H. Nakano, A. Ori and M. Sasaki, Phys. Rev. Lett. 88, 091101 (2002) arXiv:gr-qc/0111001.

[10] L. Barack and A. Ori, Phys. Rev. Lett. 90, 111101 (2003) arXiv:gr-qc/0212103.

[11] L. M. Burko, Phys. Rev. Lett. 84, 4529 (2000) arXiv:gr-qc/0003074.

[12] S. Detweiler, E. Messaritaki and B. F. Whiting, Phys. Rev. D 67, 104016 (2003) arXiv:gr-qc/0205079.

[13] An alternative algorithm for self-force calculations, also implemented in the frequency domain, has been proposed by T. S. Keidl, J. L. Friedman and A. G. Wiseman, Phys. Rev. D 75, 124009 (2007) arXiv:gr-qc/0611072.

[14] L. Barack and L. M. Burko, Phys. Rev. D 62, 084040 (2000) arXiv:gr-qc/0007033.

[15] L. Barack and C. O. Lousto, Phys. Rev. D 66, 061502 (2002) arXiv:gr-qc/0205043.

[16] R. Haas, Phys. Rev. D 75, 124011 (2007) arXiv:0704.0797 [gr-qc]].

[17] L. Barack and N. Sago, Phys. Rev. D 75, 064021 (2007) arXiv:gr-qc/0701069.

[18] J. L. Barton, D. J. Lazar, D. J. Kennefick, G. Khanna and L. M. Burko [arXiv:gr-qc/0804.1075].

[19] D. Golbourn, L. Barack and N. Sago, in preparation.

[20] G. James, Advanced Modern Engineering Mathematics (Pearson, Harlow, 2004), 3rd ed., Sec. 4.2.8.

[21] For a pedagogical introduction to the Gibbs phenomenon, see, e.g., W. J. Thompson, Am J. Phys. 60, 425 (1992).

[22] P. L. Chrzanowski, Phys. Rev. D 11, 2042 (1975).

[23] L. Barack and C. O. Lousto, Phys. Rev. D 72, 104026 (2005) arXiv:gr-qc/0510019.

[24] N. Sago and L. Barack, in preparation.

[25] C. G. Darwin, Proc. R. Soc. A 249, 180 (1959).

[26] Formally, the relevant multipoles in this case are not the standard spherical harmonics but rather the 2nd-rank tensorial harmonics. Similarly, in the discussion below regarding Teukolsky fields in Schwarzschild, the relevant multipoles are the spin-weighted spherical harmonics. These technical details do not in any way affect the discussion here.

[27] Although the full separation of the field equation in Kerr is based on the spheroidal harmonics, the mode sum method (at least in its present form [10]) is based on the spherical harmonics. Note that the latter harmonics do provide a valid mode decomposition even in Kerr, even though the field equation couples modes of different spherical-harmonic $l$. One of the possible practical ways to construct a Fourier-spherical-harmonic mode $\phi_{l m \omega}$ is by solving for the separable spheroidalharmonic modes $l^{\prime} m \omega$ for various $l^{\prime}$, and then summing over their contributions to $\phi_{l m \omega}$, as discussed in Ref. [10].

[28] Note also that the gravitational self-force calculation requires the local metric perturbation, and the construction of the latter requires one to apply certain differential operators to the Teukolsky fields $\Psi_{0}$ or $\Psi_{4}$ [22]. The situation with the EM self-force is similar, as it again requires applying certain differential operators to $\varphi_{0}$ or $\varphi_{2}$.

[29] Strictly speaking, $F_{l \mu}$ has been defined in Ref. [8] to be the $l$-multipole of $\mathcal{F}_{\mu} \Phi$, rather than $\mathcal{F}_{\mu} \Phi_{l}$. Here, however, the $\theta$ component of the self force vanishes as we consider an equatorial orbit; and for the three remaining components the two definitions coincide.

[30] Equation (23) should be viewed here as the Fourier decomposition of $\phi_{l m, r}(t, r)$, rather than the result of a term-by-term differentiation of Eq. (22). The same applies to Eq. (24).

[31] The integration limits in this expression are shifted, both by $r_{*}^{\mathrm{P}}(t=0)$, with respect to those in Eq. (D19), but this shift does not make any difference because the integration is still over a full period. 